Merci d'utiliser le titre suivant lorsque vous citez ce document :

Smith, G. (2010-01-29), «Interactions entre normes publiques et normes privées dans la filière alimentaire », Éditions OCDE, Paris.

http://dx.doi.org/10.1787/5kmmmx1h937l-fr

\title{
Interactions entre normes publiques et normes privées dans la filière alimentaire
}

\author{
Garry Smith
}

La version originale de ce document a été publiée comme suit :

Smith, G. (2009-02-01), "Interaction of Public and Private Standards in the Food Chain", OECD Food, Agriculture and Fisheries Papers, No. 15, OECD Publishing, Paris. http://dx.doi.org/10.1787/221282527214 


\section{NOTE}

Ce document relève du domaine de résultats 2 du Programme de travail et budget (PTB) 2005-2006 : Analyser l'évolution de l'économie alimentaire et, en particulier, des travaux sur les rôles respectifs des normes publiques et privées dans la réponse apportée à l'évolution de la demande des consommateurs et des attentes de la collectivité. Une version révisée du rapport a été examinée par le Groupe de travail des politiques et des marchés agricoles au cours des réunions qu'il a tenues en novembre 2007 et mai 2008. Lors de cette dernière réunion, le Groupe de travail s'est prononcé pour la déclassification du rapport. La version finale tient compte des principaux commentaires formulés à la réunion du Groupe de travail de mai 2008, qui ont été ensuite transmis par écrit. 


\section{TABLE DES MATIÈRES}

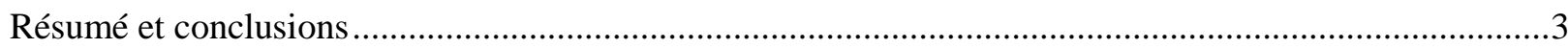

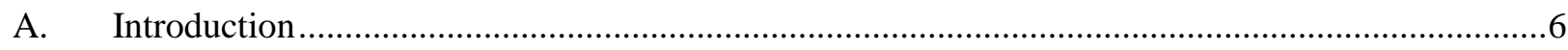

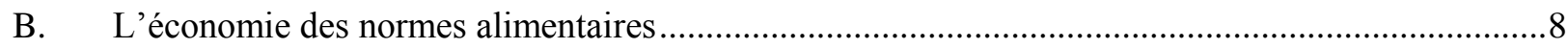

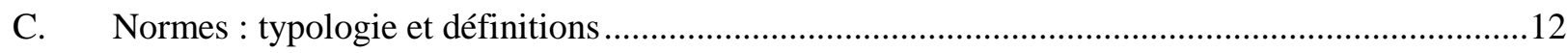

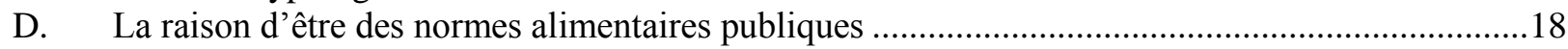

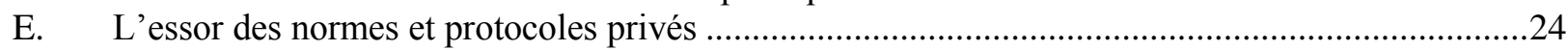

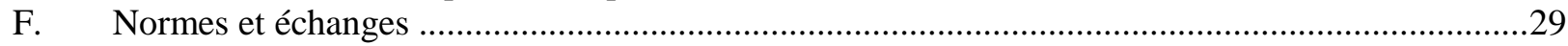

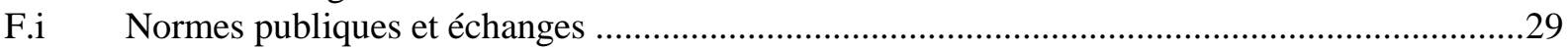

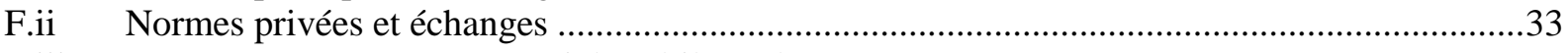

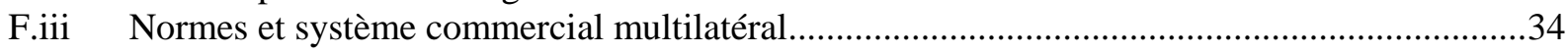

G. Quelques conclusions et domaines futurs de recherches ........................................................36

\section{Encadrés}

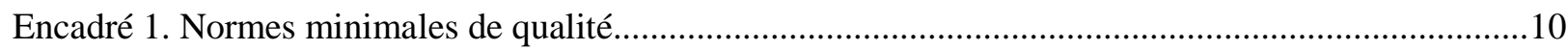

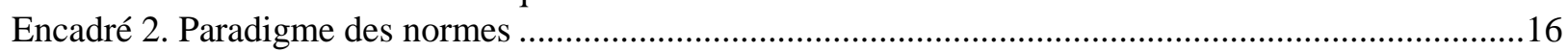




\section{INTERACTIONS ENTRE NORMES PUBLIQUES ET NORMES PRIVEES DANS LA FILIERE ALIMENTAIRE}

\section{Résumé et conclusions}

Le présent rapport passe en revue certaines des questions soulevées, principalement dans les pays développés, par le développement simultané de normes de qualité publiques et privées. Il propose une première approche des formes que prennent les interactions des normes publiques et privées de qualité des aliments dans les systèmes alimentaires modernes. Il ne s'agit pas d'analyser dans le moindre détail ce qui constitue un domaine de recherche relativement nouveau. L'examen met l'accent sur les normes alimentaires, désignant ici aussi bien l'ensemble des caractéristiques de produit et de procédé exigées par les consommateurs et la collectivité que les normes de sécurité des aliments. À l'évidence, la sécurité est un aspect incontournable de la qualité des aliments, dans la mesure où elle précède toute autre considération. Cependant, la sécurité des aliments correspond à des exigences très différentes de celles qui sont associées aux autres caractéristiques qualitatives. Elle est assimilable à un «bien public » car tout système alimentaire se doit de veiller à ce que les produits fournis inspirent confiance. On considère habituellement qu'il appartient aux pouvoirs publics d'élaborer des réglementations, le plus souvent sous la forme de normes de sécurité contraignantes, garantissant que les aliments proposés aux consommateurs sont sans danger. Compte tenu des externalités, des asymétries d'information et des caractéristiques de biens publics, les marchés ne sauraient à eux seuls apporter le degré de sécurité des aliments souhaitable pour la collectivité. Il est donc admis ici que les normes de sécurité des aliments relèvent de la compétence du secteur public et n'entrent pas dans le cadre de cet examen des interactions entre les normes de qualité des aliments publiques et privées.

Le rapport s'appuie sur une analyse des travaux antérieurs, d'où il ressort que la recherche sur les rapports et les interrelations entre normes publiques et normes privées dans les dispositifs nationaux de contrôle de la qualité des aliments et les échanges internationaux n'en est qu'à ses tout premiers balbutiements. Toutefois, à l'évidence, l'évolution des mécanismes de contrôle de la qualité des produits alimentaires s'accompagne d'interactions de plus en plus complexes entre les approches réglementaires publiques et privées. Ces dix dernières années, de nombreux pays ont sensiblement accru la surveillance officielle de la qualité des aliments, notamment en durcissant les réglementations directes, c'est-à-dire applicables en amont, et les contrôles indirects, autrement dit a posteriori. Par ailleurs, le développement rapide de protocoles et de mécanismes privés, qui tiennent une place croissante dans l'offre d'aliments de qualité, s'est traduit par la création d'un tissu de plus en plus complexe de normes publiques et privées, dont l'objectif est d'assurer la qualité grandissante des produits commercialisés sur les marchés agricoles et alimentaires.

Dans le même temps, les normes alimentaires publiques sont soumises à un examen approfondi, en particulier de la part de l'OMC, de leurs fondements scientifiques et de leur efficience économique, ce qui influe sur les évolutions en cours. Le secteur public était initialement chargé de définir des normes pour les classes, les poids et les mesures des produits utilisés dans des conditions de pleine concurrence sur des marchés de masse constitués de produits agricoles fortement homogènes. Les normes et classes en vigueur sur ces marchés, qui revêtent de nombreuses caractéristiques de biens d'intérêt public, resteront essentiellement dans le domaine public. De manière générale, les réglementations publiques portant sur la qualité des produits alimentaires sont de plus en plus fondées sur les résultats et les procédés, ce qui 
accentue la responsabilité incombant aux entreprises agroalimentaires privées de mettre en œuvre des contrôles de qualité adéquats. Sont ensuite apparues les normes de qualité privées. Destinées à atténuer les risques encourus par les entreprises agroalimentaires privées, que ce soit sur le plan réglementaire ou celui de leur réputation, elles sont de plus en plus utilisées pour faciliter le positionnement concurrentiel sur des marchés à plus forte valeur ajoutée et ce, par le biais d'une différenciation des produits fondée sur une gamme de plus en plus vaste de caractéristiques de qualité.

Parce que le progrès scientifique, l'accroissement des richesses et les nouvelles prises de conscience de la société, concernant l'environnement, le développement durable et le bien-être des animaux, confèrent un intérêt accru à un éventail plus large de caractéristiques qualitatives, les entreprises du secteur privé et les organismes publics sont de plus en plus confrontés à une demande d'amélioration de la qualité des produits alimentaires, non seulement de la part des consommateurs, mais également de l'ensemble de la collectivité. Néanmoins, comme les intérêts privés et les objectifs sociaux ne coïncident que rarement, il n'est pas certain que l'application d'un système efficace de contrôle de la qualité des aliments dans une optique commerciale permette d'obtenir de bons résultats sur le plan social. Les entreprises sont incitées à fournir des aliments de qualité pour bénéficier d'un avantage concurrentiel, mais lorsque les informations destinées aux consommateurs et permettant d'évaluer la qualité des produits alimentaires sont imparfaites, ni le marché ni les incitations légales ne suffisent à fournir aux consommateurs le niveau de qualité et de protection souhaité par la société dans son ensemble. Face aux externalités et aux asymétries d'information, le rôle des pouvoirs publics reste déterminant pour remédier aux défaillances du marché en adoptant des réglementations a minima sur la qualité des produits alimentaires. Dans le cas des biens d'expérience et des biens de confiance, les consommateurs comptent principalement sur les normes publiques et les signes d'identification pour garantir la qualité des aliments mis sur le marché. Cette situation reflète les difficultés auxquelles ils sont confrontés pour évaluer la qualité des produits alimentaires dans de tels cas ou intenter un recours contre les fournisseurs si le produit ne s'avère pas conforme à leurs attentes. Toutefois, il n'est pas impératif de recourir à des normes de qualité ou à des réglementations obligatoires dans toutes les circonstances. En effet, les pouvoirs publics peuvent également adopter des normes volontaires et tenter de les faire respecter par le biais de normes de qualité, dont le succès nécessite que les pays concernés soient prêts à imposer des normes obligatoires dès lors que les normes volontaires ne permettent pas d'atteindre un niveau adéquat de qualité des produits. Néanmoins, lorsque les pratiques des producteurs peuvent avoir de graves conséquences sur le bien-être des consommateurs, les pouvoirs publics choisissent fréquemment d'adopter des mesures préventives pour exclure ces éventualités et optent alors pour des normes obligatoires.

Lorsque des informations appropriées sur les caractéristiques de qualité d'un produit alimentaire sont mises à la disposition des consommateurs ou que ceux-ci peuvent les trouver, le rôle des normes obligatoires est davantage sujet à caution. Dans le cas des caractéristiques de recherche des qualités des produits alimentaires, le marché fournit généralement un moyen efficace d'inciter les producteurs à privilégier la qualité, et ce même en l'absence de normes volontaires ou de système adéquat de responsabilité du fait du produit. En dépit de ces arguments économiques en faveur de la mise en place de mécanismes de contrôle volontaires, il convient de noter que les dispositifs obligatoires traditionnels à caractère impératif restent prédominants dans de nombreux régimes réglementaires nationaux et régionaux.

Des divergences peuvent également exister entre les intérêts des producteurs et ceux des consommateurs dans le domaine du commerce international. S'il est dans l'intérêt des producteurs du secteur agroalimentaire d'un pays de définir des normes qui leur confèrent un avantage concurrentiel par rapport aux importations, cela ne profite pas nécessairement aux consommateurs. Cet argument paraît toutefois moins fondé dans le cas de la grande distribution et des sociétés de restauration rapide. Avec la conclusion des accords de l'OMC relatifs aux mesures sanitaires et phytosanitaires (SPS) et aux obstacles techniques au commerce (OTC), qui réglementent précisément l'application des normes par les Membres pour en atténuer les éventuels effets sur les échanges, les États font l'objet d'un examen approfondi visant 
à garantir que ces normes ne sont pas instaurées dans une optique protectionniste. Les premiers résultats issus des différends soumis à l'OMC semblent indiquer que ces procédures, en particulier dans les domaines sanitaire et phytosanitaire, parviennent dans une certaine mesure à réduire les obstacles au commerce résultant de l'instauration de normes publiques.

Les normes volontaires privées peuvent également influer sur les échanges. Dans un univers marqué par la prédominance des normes privées dans les filières d'approvisionnement mondiales contrôlées par des entreprises agroalimentaires multinationales, l'accès à la chaîne mondiale de valeur constitue une préoccupation centrale pour les exportateurs ou fournisseurs de produits alimentaires. La diminution du nombre des grands distributeurs et des multinationales de l'agroalimentaire liée au regroupement et à la concentration à l'œuvre dans le secteur accroît la perspective, pour certains exportateurs, de se voir exclus de marchés entiers en cas de manquement aux normes privées qui en réglementent l'accès. Pour les mêmes raisons, ceux qui parviennent à accéder à ces chaînes mondiales de valeur peuvent en retirer des avantages substantiels en termes de relations commerciales. Les normes peuvent ainsi servir de catalyseur pour ouvrir de nouveaux débouchés, mais le développement des normes privées ne signe pas l'abandon des normes réglementaires publiques. Cependant, pour accéder aux marchés, les exportateurs devront toujours démontrer qu'ils satisfont aux normes publiques. Cette évolution montre néanmoins qu'un renforcement de la coordination des normes publiques et des normes privées, ainsi que des mécanismes de contrôle associés, pourrait se traduire par des gains économiques. Un exemple est donné par les industries laitières des États membres de l'UE qui ont conclu un accord commun pour harmoniser les normes sur la qualité des produits laitiers au travers des États membres, accord qui a servi de base à la Commission européenne pour établir les larges normes de l'UE. Un autre exemple au niveau régional des bénéfices d'une telle coordination est donné par le Système d'Alerte Rapide dans l'UE qui a été établi en coopération avec les distributeurs et détaillants de l'industrie alimentaire privée pour pouvoir retirer rapidement les produits des étals des détaillants si des problèmes de qualité sont détectés.

Sous l'effet de la mondialisation croissante des filières d'approvisionnement alimentaires, ces deux mécanismes subissent l'influence des accords commerciaux de l'OMC et des travaux des organismes internationaux de normalisation des produits alimentaires. Si la transparence des processus réglementaires publics s'en trouve accrue, on peut néanmoins craindre que le même niveau de transparence ne puisse être appliqué aux normes privées et aux procédures de contrôle correspondantes.

La combinaison entre normes publiques et normes privées ne cesse d'évoluer. Dans les pays développés, il semble que la tendance aille dans le sens d'une dissociation entre les normes et les réglementations applicables aux produits alimentaires, les premières étant du ressort des entreprises privées et les secondes des organismes publics. Les réglementations directes accordent une plus grande importance à la responsabilité des entreprises. En outre, les normes privées, ainsi que les forces du marché liées à la segmentation des marchés et à l'amélioration de la réputation d'une entreprise qui en découle tendent à conforter cette évolution. Ce transfert des responsabilités au secteur privé se traduit par un espace d'intervention plus complexe associant incitations et contrôles du secteur public et du secteur privé. Dans ce cas, il faut que les instances réglementaires publiques situent mieux leurs actions par rapport à l'évolution des modes privés de gestion de la qualité alimentaire, car les modalités d'élaboration des normes publiques peuvent accroître les incitations marchandes à investir dans le contrôle des filières d'approvisionnement et de la qualité des aliments.

À en juger par cette brève analyse des travaux antérieurs, les normes de qualité alimentaire privées sont souvent plus sévères, tout en offrant davantage de souplesse et de possibilités d'adaptation aux goûts des consommateurs qui ne cessent d'évoluer, que les normes de qualité publiques appliquées à l'initiative de différents pays pour leur propre approvisionnement alimentaire ou à la suite de négociations internationales (Codex); dans l'ensemble, les normes publiques et privées de qualité alimentaire tendent à se compléter. Dans de nombreux cas, les normes privées s'appuient sur l'infrastructure existante des 
normes publiques pour fournir un élément de concurrence par la différentiation de qualité. En effet, les normes publiques demeurent nécessaires pour remédier aux défaillances du marché découlant d'asymétries d'information ou d'externalités de consommation et lorsque les normes ont à l'évidence des caractéristiques de bien d'intérêt public. Elles conserveront ainsi un rôle prédominant dans l'instauration de classes et de normes de base, non seulement pour permettre aux marchés de masse caractérisés par des productions agricoles particulièrement homogènes de générer des économies d'échelle, mais aussi pour garantir aux consommateurs des normes de sécurité alimentaire minimales, empêcher la fraude ou la tromperie sur la qualité du produit. Les normes publiques jouent également un rôle au niveau mondial, où elles assurent la cohérence des normes de base entre les différents pays et leur adéquation avec les normes à caractère obligatoire (SPS, OTC) dans les échanges transfrontières. En l'absence de normes publiques efficaces, les normes privées peuvent s'y substituer pour garantir la qualité des aliments commercialisés. Dans d'autres cas, des normes privées ont été mises en place pour différencier les produits alors même qu'existaient des normes publiques rigoureuses, ce qui montre à quel point la qualité est un atout concurrentiel de plus en plus important, non seulement sur les marchés agricoles et alimentaires, mais aussi pour améliorer l'efficacité de la coordination des filières d'approvisionnement régies par les acheteurs, qui se développent de plus en plus. Sur des marchés de produits différenciés et pour répondre aux exigences de certains marchés de niche (notamment en termes de responsabilité du fait des produits), les entreprises agroalimentaires peuvent choisir d'instituer des normes privées plus contraignantes que les normes publiques. Les normes privées et normes publiques peuvent alors se renforcer mutuellement et améliorer l'efficacité globale du système, ce qui accroîtra la qualité des aliments disponibles sur les marchés nationaux et mondiaux.

\section{A. Introduction}

Les normes, publiques et privées, sont de plus en plus omniprésentes dans les systèmes agroalimentaires contemporains. Elles font désormais partie intégrante des échanges tout au long de la chaîne d'approvisionnement des produits agricoles transformés, sans se limiter aux produits bruts [Henson et Reardon, 2005]. Les pouvoirs publics sont habituellement les mieux placés pour instaurer des normes et règles de qualité alimentaire minimales dans l'intérêt de la population. Autrement dit, il leur appartient dans une certaine mesure de veiller à la qualité des aliments, pour assurer, entre autres choses, l'accès de tous à une alimentation sûre ${ }^{1}$ et protéger les consommateurs contre des pratiques abusives et frauduleuses.

Les normes ne cessent de s'adapter à l'évolution des technologies et des connaissances scientifiques sur les risques alimentaires et, de façon directe, aux demandes émanant des consommateurs et de la collectivité. Elles se sont peu à peu multipliées et étendues à des domaines plus divers du fait de la complexité grandissante des activités en jeu. Parallèlement, dans beaucoup de pays développés, des changements structurels et institutionnels liés à des systèmes de contrôle et dispositifs coercitifs privés se font jour dans l'agroalimentaire, tandis que certaines tendances de la demande des consommateurs

1 À l'évidence, la sécurité est un aspect incontournable de la qualité des aliments, dans la mesure où elle précède toute autre considération. Cependant, la sécurité des aliments correspond à des exigences très différentes de celles qui sont associées aux autres caractéristiques qualitatives. Elle est assimilable à un bien public » car tout système alimentaire se doit de veiller à ce que les produits fournis inspirent confiance. On considère habituellement qu'il appartient aux pouvoirs publics d'élaborer des réglementations, le plus souvent sous la forme de normes de sécurité contraignantes, garantissant que les aliments proposés aux consommateurs sont sans danger. Compte tenu des externalités, des asymétries d'information et des caractéristiques de biens publics, les marchés ne sauraient à eux seuls apporter le degré de sécurité des aliments souhaitable pour la collectivité. Il est donc admis ici que les normes de sécurité des aliments relèvent de la compétence du secteur public et n'entrent pas dans le cadre de cet examen des interactions entre les normes de qualité des aliments publiques et privées. 
renforcent le rôle des normes volontaires privées dans la chaîne alimentaire. La généralisation des normes privées impose aux filières d'approvisionnement de délaisser la concurrence axée sur les prix au profit d'une concurrence axée sur la qualité [Henson et Reardon, 2005]. Les normes de qualité sont de plus en plus souvent considérées comme des biens privés qui différencient les produits alimentaires et relèvent de plus en plus des entreprises privées. Par ailleurs, les normes et les contrôles réglementaires du secteur public ont progressivement évolué dans la plupart des pays, dans le sens de la rigueur et de la complexité, du fait que les consommateurs recherchent des caractéristiques spécifiques ou exigent d'être informés sur certaines caractéristiques des aliments [Josling et al., 2004].

Les filères d'approvisionnement alimentaire transcendent de plus en plus les frontières nationales, notamment grâce à de nouveaux produits transformés et à un cadre d'action plus favorable aux échanges internationaux. Les normes minimales de qualité protègent les consommateurs, mais elles empêchent souvent les acteurs de la distribution, de la restauration et de la transformation, dans le système alimentaire actuel, de différencier qualitativement leurs produits pour préserver ou augmenter leurs parts de marché alors qu'ils doivent soutenir la concurrence sur les marchés nationaux et régionaux ${ }^{2}$. Des normes privées sont donc apparues pour combler ces lacunes et suivre les évolutions réglementaires. En général, elles instaurent des systèmes coordonnés qui visent à répondre à la demande des consommateurs concernant des caractéristiques qualitatives en s'appuyant sur la différenciation des produits et sur la segmentation du marché [Fulponi, 2006]. À mesure que les groupes privés et les détaillants du secteur agroalimentaire étendent leurs activités et mettent en place des filières d'approvisionnement locales et mondiales, ils définissent des normes de qualité pour les produits qu'ils achètent en amont et vendent aux consommateurs. Ces normes peuvent être plus strictes et plus exigeantes que les normes minimales imposées par les pouvoirs publics sur les marchés nationaux. Toutefois, il s'agit d'un phénomène relativement récent et les normes privées ne sont pas encore appliquées partout, si bien que les normes publiques demeurent la principale forme de contrôle dans les systèmes alimentaires d'un certain nombre de pays.

Dans ces conditions, beaucoup de questions se posent sur les relations qu'entretiennent normes publiques et normes privées au sein du système alimentaire moderne. Compte tenu de l'importance croissante des normes volontaires privées, souvent plus exigeantes, plus fréquemment ajustées et applicables à des domaines plus variés que les normes publiques, quel est le rôle de ces dernières et des réglementations alimentaires ? Peut-on s'en remettre aux décisions des sociétés privées pour obtenir des résultats conformes aux objectifs publics ? Question corollaire : les dispositifs actuels d'élaboration des normes contribuent-ils à harmoniser les résultats obtenus par les secteurs public et privé ? Les normes publiques ont-elles toujours une utilité, et laquelle ? Les normes privées seraient-elles moins exigeantes en l'absence de normes publiques de qualité alimentaires minimales?

Il s'agit ici d'éclaircir ces questions à l'interface entre normes alimentaires publiques et privées à partir de quelques exemples tirés de l'abondante documentation disponible. L'accent est mis sur le rôle joué par les normes publiques et privées dans les systèmes alimentaires actuels, l'évolution des liens qui les unissent et la manière dont elles interagissent (qu'elles se complètent, se substituent les unes aux autres, entrent en conflit ou, au contraire, convergent) pour inciter les fournisseurs à approvisionner les marchés nationaux et internationaux en produits alimentaires de bonne qualité.

Le rapport s'articule comme suit: la section B passe en revue les notions économiques pertinentes, dont découlent la typologie et les définitions des normes alimentaires dans la section C. La section D porte sur la raison d'être des réglementations ou normes publiques applicables aux produits alimentaires. Les facteurs expliquant l'apparition et l'importance croissante des normes volontaires privées sont évoqués

2 Les catégories et les normes datent bien souvent d'une époque marquée par des marchés de produits agricoles non transformés et des biens relativement homogènes. 
dans la section E. La section F inscrit la réflexion sur les normes dans le contexte plus large des échanges internationaux. Une dernière section vise à dégager des enseignements sur les différents rôles et interactions des normes publiques et privées dans les systèmes alimentaires nationaux et internationaux.

\section{B. L'économie des normes alimentaires}

La documentation économique sur les normes, telles qu'elles étaient initialement conçues, a mis en avant la fourniture d'informations et la compatibilité, c'est-à-dire l'approche par les externalités de réseau, dans le processus de normalisation [Katz et Shapiro, 1985]. Les gains économiques augmentent grâce aux effets positifs de réseau, qui se font jour lorsque la valeur d'un bien s'accroît, pour l'utilisateur, à mesure que le nombre d'utilisateurs optant pour le même bien ou un bien compatible s'élève [Becker, 1999]. Les téléphones ou les fax sont des exemples classiques d'externalités de réseau positives, puisque leur valeur pour un individu augmente avec le nombre d'utilisateurs reliés à ce service. Les travaux économiques en matière de normalisation alimentaire ont surtout envisagé le rôle des normes publiques de qualité des aliments comme instruments de réglementation des marchés de produits alimentaires et comme mesures non tarifaires possibles, bien qu'une plus grande attention soit désormais accordée à l'importance croissante des normes de qualité privées dans la chaîne d'approvisionnement alimentaire [Henson, 2006].

L'économie des normes est envisagée ici sous l'angle de la qualité des produits alimentaires, et ce pour plusieurs raisons. Premièrement, l'augmentation des revenus et l'élévation du niveau de vie aidant, les consommateurs sont généralement devenus plus exigeants et moins uniformes dans leurs choix alimentaires. Ainsi s'explique en partie l'apparition de normes privées, la concurrence entre les entreprises étant désormais plus fondée sur la différenciation qualitative des produits que sur les prix des produits alimentaires. Deuxièmement, les produits agroalimentaires sont très variés et hétérogènes, ce qui conduit les consommateurs à s'interroger sur les différentes caractéristiques qui composent la qualité des aliments. Du fait que la qualité dépend aussi bien des caractéristiques objectives des produits que des préférences subjectives des consommateurs, elle est difficile à définir en tant que telle ${ }^{3}$. Deux conceptions analytiques différentes ont été retenues dans les études consacrées à la qualité des aliments ${ }^{4}$. Les travaux sur le comportement des consommateurs privilégient une approche fondée sur les qualités ou caractéristiques perçues par le consommateur, tandis que les études scientifiques sur l'alimentation s'intéressent davantage à la mesure de la qualité sur la base de propriétés objectives [Bowbrick, 1992]. Selon la première approche, qui est celle de Lancaster [1966], la qualité est envisagée comme un faisceau d'attributs, de caractéristiques ou de propriétés qui déterminent les performances d'un produit. Les caractéristiques qualitatives des produits alimentaires relèvent de plusieurs grandes catégories : aliments, valeur nutritionnelle, propriétés organoleptiques (couleur ou aspect, saveur, texture, arôme et autres caractéristiques sensorielles, etc.) et caractéristiques liées aux procédés, englobant la production biologique, le bien-être animal, l'absence d'OGM, la protection de l'environnement et les conditions de travail [Brunsø et al., 2002].

On suppose que les consommateurs s'en remettent à certains indices et signaux pour repérer les attributs qu'ils recherchent [Becker, 1999]. Une distinction est généralement établie entre indices

3 L'Organisation internationale de normalisation (ISO) a donné de la qualité alimentaire une définition largement répandue, à savoir «l'ensemble des propriétés et des caractéristiques d'un produit, qui lui confèrent l'aptitude à satisfaire des besoins exprimés ou implicites ». Néanmoins, cette définition est si générale qu'elle n'est pas utilisable à des fins analytiques.

$4 \quad$ Ces deux approches sont potentiellement contradictoires, comme on le voit au travers du différend qui oppose les États-Unis à l'Union européenne sur l'affaire du bœuf aux hormones. Alors que l'UE est favorable à l'approche des qualités perçues, les Etats-Unis font valoir qu'aucune preuve scientifique ne confirme la perception des consommateurs selon laquelle le bœuf serait d'une qualité moindre lorsqu'il est traité aux hormones de croissance. D'après eux, aucune raison ne justifie donc l'embargo européen sur les importations de bœuf en provenance des États-Unis, qui constitue une violation des règles de l'OMC. 
intrinsèques et indices extrinsèques. Les premiers renvoient aux propriétés physiques d'un produit alimentaire, le reste correspondant pour l'essentiel à des indices extrinsèques [Grunert, 2005]. Des indices intrinsèques comme la couleur, l'aspect, la taille et l'arôme renseignent sur des attributs tels que la qualité gustative ou la saveur. Parmi les divers indices extrinsèques, trois ont plus particulièrement retenu l'attention en raison du signal qualitatif qu'ils peuvent donner aux consommateurs : les marques, les indications d'origine et les labels de qualité. Normalement, les consommateurs choisissent les produits en fonction de l'ensemble de leur alimentation, dans une optique d'utilité. Selon leurs préférences vis-à-vis du risque, ils s'orientent vers différents ensembles d'aliments. Si leurs attentes qualitatives ne sont pas satisfaites (risques sanitaires ou autres associés à certains aliments, par exemple), les consommateurs subissent une perte d'utilité. Du côté de l'offre, les producteurs fournissent des aliments de qualité s'ils y gagnent ou s'ils y sont contraints. Un gain plus élevé peut être enregistré par ceux qui misent sur la différenciation et vendent leurs produits sur des marchés spécialisés, ou qui évitent les pertes liées à des procédures coûteuses et le versement de dommages-intérêts si l'engagement de qualité n'est pas tenu. Autrement dit, pour la qualité comme dans d'autres domaines, le résultat sur le marché est déterminé par l'offre et la demande de certaines caractéristiques, ainsi que par les informations dont disposent les consommateurs sur ces caractéristiques [Caswell et Mojduszka, 1996].

Généralement, le marché de la qualité alimentaire peut être décrit par une courbe ascendante de l'offre (coût marginal) et une courbe descendante de la demande (bénéfice marginal) [Henson et Traill, 1993 ; Caswell et Mojduszka, 1996]. Dans des conditions de marché parfaites, ces deux courbes se rejoignent au prix d'équilibre, qui correspond à la qualité alimentaire optimale. Ce scénario suppose que tous les acteurs du marché sont pleinement informés de la nature du produit proposé, qu'acheteurs et vendeurs sont essentiellement des preneurs de prix, et que les prix du marché tiennent compte de l'ensemble des coûts et avantages pour la collectivité. Dans cette hypothèse, les prix du marché transmettent toutes les informations utiles, il n'apparaît pas d'externalités, et aucune intervention des pouvoirs publics n'est nécessaire [Caswell et Mojduszka, 1996]. Un éventail de produits de qualités différentes sera alors proposé sur le marché à différents prix. Or le marché de la qualité alimentaire est souvent loin d'être parfait. Les imperfections les plus graves proviennent de l'asymétrie d'information : les vendeurs sont généralement mieux informés sur la qualité que les acheteurs. En outre, étant donné qu'il est souvent difficile, voire impossible, pour les acheteurs de savoir si les produits sont de bonne ou de mauvaise qualité avant l'achat (biens de confiance), les vendeurs sont incités à faire des promesses tout en vendant des produits de qualité inférieure, d'où une sélection négative mise en évidence par Akerlof [1970]. Les consommateurs peuvent également se faire une idée erronée des risques associés à la consommation de certains aliments (externalités), et l'information sur la qualité alimentaire peut présenter les caractéristiques d'un bien public. Dans ce cas, l'offre de qualité alimentaire peut être trop forte ou trop faible [Caswell et Mojduszka, 1996]. 


\begin{tabular}{|c|c|}
\hline & Encadré 1. Normes minimales de qualité \\
\hline & $\begin{array}{l}\text { A l'échelle mondiale, le rôle économique de l'information est un élément fondamental à la compréhension de } \\
\text { nportance des normes au sein du système alimentaire. De manière générale, les normes peuvent améliorer les } \\
\text { ormations apportées au consommateur sur la qualité des produits. Sa confiance s'en trouve renforcée, ce qui } \\
\text { traîne une progression générale de la demande. Les normes minimales peuvent être utilisées dans les situations } \\
\text { nformation imparfaite. Sur un plan théorique, les spécialistes considèrent les "normes minimales de qualité " } \\
\text { mme un minimum pouvant être appliqué à n'importe quelle caractéristique, le raisonnement économique demeurant } \\
\text { changé. }\end{array}$ \\
\hline & $\begin{array}{l}\text { En cas d'asymétrie d'information, les organismes de réglementation peuvent définir des normes minimales de } \\
\text { Ialité. Le plus souvent, ces normes sont utilisées dans le but d'améliorer la qualité des biens produits et consommés, } \\
\text { oique le débat sur leurs retombées finales soit loin d'être clos. Lorsque les pouvoirs publics établissent des normes } \\
\text { nimales de qualité, c'est habituellement pour des raisons liées aux externalités de consommation, lorsque la qualité } \\
\text { s produits présents sur le marché libre est jugée insuffisante. L'adoption de normes minimales de qualité pour } \\
\text { rtaines caractéristiques de produits ou de procédés réduit les différences de qualité entre les entreprises (puisque } \\
\text { ut produit dont la qualité est inférieur au minimum requis n'est pas autorisé à être vendu) et renforce la concurrence } \\
\text { Ir les prix. Les entreprises peuvent alors décider d'adopter des normes de qualité plus strictes pour atténuer la } \\
\text { ncurrence par les prix. Cependant, comme les différences qualitatives ne retrouvent jamais tout à fait leur amplitude } \\
\text { tiale, les prix baissent, ou augmentent dans une moindre mesure, et le bien-être s'accroît [Ronnen, } 1991 \text {; Gal-Or, } \\
\text { 97)] }]^{1} \text {. Selon Leland [1979] et Shapiro [1983], entre autres, les normes minimales de qualité réglementaires ne } \\
\text { ffisent pas à améliorer la qualité pour les consommateurs car beaucoup ont déjà accès à un niveau dépassant celui } \\
\text { s normes minimales, tandis que d'autres peuvent se voir exclus du marché par la hausse des prix. }\end{array}$ \\
\hline & $\begin{array}{l}\text { Dans le secteur alimentaire, les normes minimales de qualité sont très utilisées pour les produits et procédés } \\
\text { s à des caractéristiques de consommation telles que biologiques ou fermières }{ }^{2} \text {. Pour asseoir et maintenir leur } \\
\text { putation, ou pour fidéliser leur clientèle et dégager des bénéfices, les distributeurs peuvent décider de fixer des } \\
\text { veaux de qualité supérieurs aux normes minimales. Pour certains, ce comportement constitue une réponse à la } \\
\text { mande des consommateurs souhaitant une meilleure qualité et disposés à en payer le prix [Lutz et al., } 2000 \text {; } \\
\text { chia et Lambertini, } 1997 \text {; Boom, } 1995 \text {; Crampes et Hollander, 1995]. Cette demande explique également } \\
\text { tablissement, dans la distribution de détail, de gammes spécifiques de produits de qualité supérieure, comme le } \\
\text { ature's choice de Tesco, la Filière de Qualité de Carrefour ou le President's choice de Loblaw. Une augmentation de } \\
\text { mise en place de normes privées plus strictes que les normes minimales de qualité soulève la question de la } \\
\text { otivation économique ou stratégique des entreprises. S'agit-il d'un nouveau mode de concurrence, d'une manière de } \\
\text { prémunir contre les éventuelles défaillances de qualité des produits, d'une stratégie d'anticipation des } \\
\text { glementations publiques ou, tout simplement, d'une volonté d'influencer les réglementations futures? }\end{array}$ \\
\hline & $\begin{array}{l}\text { Une des stratégies possibles consiste à anticiper sur les réglementations publiques. Les entreprises, } \\
\text { dividuellement ou en groupes, peuvent décider d'adopter des normes plus exigeantes que les normes existantes si, } \\
\text { faisant, elles peuvent influer sur les réglementations à venir. La synchronisation des décisions prises par les } \\
\text { uvoirs publics et par les entreprises a, ici encore, son importance [Lutz et al., 2000]. Si les entreprises décident de } \\
\text { isser à une norme plus exigeante avant que les autorités publiques ne le fassent, les instances de réglementation } \\
\text { uvent être amenées à définir des normes minimales moins strictes que dans d'autres circonstances. Dans ce cas de } \\
\text { ure, les entreprises agroalimentaires peuvent aussi avoir consenti des investissements substantiels en machines, } \\
\text { uipement ou d'autres aspects logistiques afin de garantir l'adoption et l'application de leur propre norme privée. Or, } \\
\text { s pouvoirs publics peuvent ne pas souhaiter augmenter les coûts supportés par les entreprises, compte tenu des } \\
\text { nséquences néfastes que cela peut avoir sur les prix ou sur l'emploi, en instaurant des normes minimales } \\
\text { férentes des leurs. Néanmoins, cette stratégie d'anticipation peut restreindre le bien-être social par rapport au } \\
\text { veau qu'il aurait atteint si les pouvoirs publics avaient agi les premiers [Lutz et al., 2000]. Il peut en aller ainsi lorsqu'il } \\
\text { existe encore aucune norme minimale de qualité. Dans le secteur alimentaire, ce phénomène pourrait concerner les } \\
\text { ractéristiques de procédés, en particulier le bien-être des animaux et la protection de l'environnement. Dans certains } \\
\text { s, les entreprises surenchérissent sur les normes en place de façon à consolider leur réputation de fournisseurs de } \\
\text { oduits de grande qualité sur des marchés de niche. Dans la mesure où cette surenchère peut conforter la réputation } \\
\text { une entreprise et les recettes qu'elle tirera des ventes sur le marché, la publicité de son comportement peut l'inciter à } \\
\text { faire davantage [Lutz et al., } 2002 \text {; Boom, 1995]. }\end{array}$ \\
\hline & $\begin{array}{l}\text { Ces résultats sont obtenus sur des marchés en concurrence imparfaite, où les vendeurs peuvent davantage fixer les prix qu'agir en preneurs (voir } \\
\text { Leland et Shapiro). Ronnen estime que la concurrence menée sur le terrain de la qualité dessert la concurrence par les prix: pour atténuer les } \\
\text { effets d'une intensification de cette concurrence sur leur chiffre d'affaires, les entreprises vendant des produits de qualité améliorent encore celle-ci } \\
\text { en réponse au relèvement du niveau qualitatif opéré par les entreprises vendant des produits de moindre qualité pour respecter les normes } \\
\text { minimales de qualité en vigueur. Par conséquent, si les coûts variables n'augmentent pas « trop rapidement " avec l'amélioration de la qualité, les } \\
\text { prix corrigés en fonction de la qualité baissent [Ronnen, } 1991, \text { p. 503]. } \\
\text { Lorsque les normes minimales sont établies de façon exogène, elles peuvent ne pas être optimales. Elles le sont en revanche quand l'équilibre a } \\
\text { été trouvé entre les avantages échus aux consommateurs de biens de grande qualité et les pertes subies par ceux qui auraient préféré acheter un } \\
\text { produit d'une qualité inférieure à la norme minimale. Les organismes de réglementation mettent en place des normes minimales pour les biens dont } \\
\text { les caractéristiques pourraient avoir des répercussions négatives. }\end{array}$ \\
\hline
\end{tabular}


Les économistes ont souvent classé les biens en trois catégories, selon les informations dont disposent les consommateurs lors de leur achat. La première correspond aux biens de recherche. Dans le cas des biens ou des caractéristiques de recherche, les consommateurs sont en mesure de vérifier visuellement les propriétés d'un produit avant de l'acheter et de le consommer, en l'examinant ou en se renseignant à son sujet. Il n'existe pas d'incertitude qualitative, étant donné l'inspection minutieuse préalable à l'achat, dans l'hypothèse d'un coût de recherche négligeable. On peut parler d'une situation d'information parfaite. S'agissant des biens ou caractéristiques d'expérience, les consommateurs ne peuvent déterminer la qualité du produit qu'après l'avoir acheté et consommé. En cas d'achats répétés, la caractéristique d'expérience peut permettre d'éliminer toute incertitude concernant la qualité qui pourrait subsister dans l'esprit du consommateur [Nelson, 1970]. Les biens de confiance sont ceux dont les caractéristiques qualitatives importent au consommateur mais restent indiscernables, même après consommation [Darby et Karni, 1973 ; Andersen et Philipsen, 1998]. En pratique, un même produit alimentaire peut présenter des caractéristiques relevant des trois catégories. Par exemple, le choix d'une tomate sur un étal de fruits et légumes peut faire intervenir la « recherche » d'une tomate en apparence mûre, au parfum agréable, ne présentant aucun signe extérieur de maladie ou d'attaque d'insecte. Après avoir mangé la tomate, fort de son «expérience », le consommateur en apprécie la qualité en fonction de divers facteurs subjectifs, tels que la saveur, la fermeté et la texture. Toutefois, les caractéristiques de «confiance » lui échappent. Elles peuvent se rapporter à la méthode de production employée, obéissant par exemple aux principes de l'agriculture biologique, ou à la présence d'éléments ou de résidus toxiques susceptibles d'avoir un effet cumulatif à long terme sur la santé. Autrement dit, le consommateur se contente de la conviction que ces caractéristiques s'appliquent ou font défaut, selon le cas, à la tomate consommée. Les caractéristiques de confiance peuvent être particulièrement problématiques, dès lors que le consommateur n'a aucun moyen d'en vérifier l'existence, ni en procédant à une recherche avant l'achat (dont le coût serait prohibitif), ni en se fondant sur l'expérience après la consommation.

Les marchés des caractéristiques de recherche vont généralement de pair avec des transactions simples, telles que les opérations de troc ou les ventes sur les marchés de plein air. Étant donné que les consommateurs ont accès à toutes les informations, ces marchés peuvent assurer efficacement la fourniture de biens de recherche, et ne nécessitent guère, voire pas du tout, d'intervention directe des pouvoirs publics. En revanche, les marchés de biens d'expérience ou de confiance ne fonctionnent pas sans élément de confiance externe. Pour les caractéristiques d'expérience, l'information est cruciale car c'est elle qui détermine ce que les consommateurs peuvent apprendre sur la qualité des produits. Sur ces marchés, les producteurs tendent à détenir plus d'informations qualitatives que les consommateurs. Les entreprises peuvent alors en profiter pour vendre des produits de qualité médiocre à un prix qui s'appliquerait à des produits de meilleure qualité plus coûteux à produire. Elles risquent toutefois de perdre leur clientèle, et des recettes, car les consommateurs se rendront ailleurs pour procéder aux mêmes achats à l'avenir. Les marchés de biens d'expérience ont donc la capacité de s'auto-corriger, puisque la demande peut réagir au fil du temps à l'évolution de la qualité des produits, surtout s'il s'agit de transactions répétées. Ceux qui correspondent à des achats peu fréquents ont souvent besoin d'être accompagnés par d'autres structures pour que la transaction prenne effet (marques, garanties ou certification, par exemple). À long terme, le consommateur disposera d'une information qualitative presque parfaite.

Le problème est plus délicat dans le cas des biens de confiance. Pour ces derniers, le consommateur manque d'information sur la qualité des produits, faute de pouvoir discerner les attributs de confiance avant l'achat, mais aussi après consommation répétée des aliments. Les modèles qui se réfèrent à des consommateurs informés et à la réputation des distributeurs sur les marchés pour les caractéristiques d'expérience se prêtent mal à des caractéristiques de confiance comme la sécurité alimentaire,la qualité nutritionnelle et les méthodes de production. Une asymétrie d'information entache également les marchés des biens de confiance dans le domaine de l'alimentation, puisque les consommateurs en savent moins que les distributeurs sur les caractéristiques qualitatives des produits. Bien que des transactions soient possibles sur des marchés non réglementés de biens de confiance, les connaissances imparfaites des consommateurs 
compromettent sérieusement les perspectives de résultats économiquement efficients. Beaucoup de produits alimentaires présentent des éléments qui relèvent à la fois de l'expérience et de la confiance. Par conséquent, on peut considérer que les produits agricoles et alimentaires forment un ensemble complexe de caractéristiques qualitatives, selon des agencements et des dosages différents, dont beaucoup ne sont pas directement observables au moment de l'achat ou même après la consommation [Henson, 2006]. Pour fonctionner correctement, ces marchés ont besoin d'une structure réglementaire publique ou d'un signal de qualité privé pour pallier l'incertitude du côté du consommateur.

Les caractéristiques de la qualité alimentaire relevant de la confiance, notamment dans le cas des produits «biologiques» ou du «commerce équitable», ne sont habituellement pas considérés être des biens publics. Sont visées des caractéristiques pour lesquelles certains consommateurs consentent à payer davantage, dès lors qu'ils peuvent en vérifier la présence par le biais d' « indications de qualité » qui lèvent les incertitudes liées au choix. Face à la défaillance du marché résultant de l'asymétrie d'information, la solution consiste essentiellement à informer davantage de manière à remédier au problème de la sélection négative. Les labels de qualité, les marques, les garanties et la certification sont autant de moyens d'informer les consommateurs en réduisant l'incertitude. D'après Caswell [1998], la labellisation peut aider les consommateurs à passer de la confiance à la recherche, en leur permettant d'apprécier la qualité du produit alimentaire avant l'achat. Encore faut-il, pour réduire véritablement l'asymétrie et permettre aux consommateurs de faire des choix optimaux conformément à leurs préférences, que cette information complémentaire soit assimilée et utilisée par le public visé. Des informations pléthoriques, ou qui ne s'adressent pas expressément aux consommateurs, risquent de provoquer une saturation et l'indifférence des consommateurs [Verbeke, 2005]. L'efficacité d'une indication de qualité dépend essentiellement de deux conditions : la pertinence de l'information apportée et sa crédibilité pour le consommateur auquel elle s'adresse [Valceschini, 1998]. Pour les biens de confiance, les normes publiques ne sont pas le seul moyen, ni nécessairement le meilleur, de remédier aux problèmes d'information sur la qualité. Une telle évaluation doit cependant être effectuée au cas par cas, en règle générale. Les consommateurs peuvent se référer aux allégations volontaires ou aux garanties des producteurs, encadrées dans certains cas par la législation de façon à éviter les allégations mensongères. Il est aussi possible, par une norme publique, d'obliger à faire connaître aux consommateurs les caractéristiques des produits ou des procédés de production. Les consommateurs peuvent également s'en remettre à la certification par tierce partie, émanant d'un organisme indépendant, pour juger de la fiabilité des informations sur la qualité souhaitée des caractéristiques des aliments [Ippolito, 2003]. Soit un certificat de conformité est établi par une tierce partie indépendante, soit la norme publique est assortie de vérifications et d'inspections de conformité. Cependant, tout en remédiant à une défaillance du marché intérieur, les certificats et labels peuvent aussi avoir des effets transfrontières dont il sera question plus loin.

\section{Normes : typologie et définitions}

Les normes existent dans tous les champs de l'activité humaine et visent à la fois à simplifier les transactions et à conférer un élément de certitude à l'échange ${ }^{5}$. Elles remplissent un certain nombre de fonctions diverses. Dans le domaine technique, les normes réduisent les risques, renforcent la crédibilité et la confiance, et contribuent à la prévisibilité pour les acheteurs et les vendeurs. Elles sont donc nécessaires au bon déroulement des échanges entre agents économiques anonymes, puisque les parties à une transaction doivent être assurées de la nature et de la qualité du produit concerné. En réduisant les coûts de recherche et de transaction et en diminuant les risques, les normes facilitent l'échange et le commerce. L'efficacité de l'échange est améliorée grâce à deux des fonctions de la norme - garantie d'une qualité minimale et définition des caractéristiques ou spécifications du produit ou de son procédé de fabrication, ainsi que des critères de performance associés. Bien que la normalisation soit nécessaire pour faciliter les

\footnotetext{
5

Une norme peut être définie comme une spécification ou un ensemble de spécifications relatives à certaines
} caractéristiques d'un produit ou de sa fabrication (Sykes, 1990). 
opérations sur le marché et implique un certain degré d'homogénéité entre produits, la variété n'est pas pour autant à rejeter. Les normes correspondent aux besoins des groupes qui les expriment et, tant que ces groupes diffèrent, leurs normes optimales reflètent ces différences [Casella, 2001].

D'après Jones et Hill (1994) et Reardon et Farina (2001), les catégories et les normes recouvrent un ensemble d'exigences techniques, définitions, conditions et principes de classification et d'étiquetage ${ }^{6}$. Elles comprennent des dispositions relatives au poids ou aux dimensions d'un produit, fixées par la réglementation ou les autorités compétentes (normes) et un système de classification fondé sur des attributs quantifiables (catégories). Les normes et catégories relatives aux «produits » alimentaires, par opposition à celles qui concernent les "procédés», spécifient les caractéristiques du produit final. Parmi ces caractéristiques peuvent figurer la forme, la taille, le poids, la sécurité, l'authenticité, la valeur énergétique, la teneur nutritionnelle ou des caractéristiques organoleptiques de qualité (couleur/apparence, saveur, texture, etc.). Les normes et catégories de produits visent à faciliter le fonctionnement des systèmes de commercialisation agricole et alimentaire. Toutefois, dans une économie agricole et alimentaire de plus en plus mondialisée, elles perdent leur rôle traditionnel ou historique de réduction des coûts de transaction sur les marchés de masse pour devenir des instruments stratégiques de différenciation des produits et de segmentation des marchés [Clayton et al., 2003]. Les normes de produits, par nature, jouent un rôle sur les marchés de produits différenciés verticalement, c'est-à-dire existant en différentes variétés et pouvant être classés selon une échelle objective donnée (classement du lait en fonction de la teneur en matière grasse, par exemple). La notion de norme minimale, utilisée pour des biens différenciés verticalement, signifie que seuls les produits atteignant ou dépassant un certain niveau de qualité sont réputés satisfaire à la norme.

Par opposition aux normes de «produits », les normes de «procédés » définissent les caractéristiques des opérations entrant dans la production des aliments : production biologique, respect du bien-être animal, procédés ménageant l'environnement, conditions de travail, commerce équitable, etc. Les normes de procédés précisent les techniques de production à respecter, du produit brut à la transformation et à l'obtention du produit intermédiaire jusqu'au produit fini prêt à être emballé et distribué. Les normes de procédés sont adoptées pour des raisons diverses : parce qu'elles influent sur les produits fabriqués (normes d'hygiène), sur l'efficacité du processus de production (externalités de réseau) ou sur l'environnement (normes de pollution) [OMC, 2005]. Les normes de «résultats» définissent les caractéristiques qu'un produit est censé présenter lorsqu'il atteint un certain point dans la chaîne agroalimentaire, par exemple la quantité de résidus de pesticides admise pour les pommes achetées aux producteurs par les transformateurs [Reardon et Farina, 2001].

Selon Valceschini et Saulais (2005), quatre grands critères de comparaison peuvent être appliqués à un système de normalisation. Ils sont mis en évidence dans le tableau 2 de l'encadré 2 . Ces critères concernent l'origine de la norme (qui la créée), la fonction ou le contenu de la norme, les modalités de mise en œuvre de la norme et la méthode de contrôle ou de vérification de la conformité. Les normes de qualité alimentaire peuvent être envisagées séparément selon qu'elles sont privées ou publiques, bien que la distinction ne soit pas toujours évidente. Dans bien des cas, des normes adoptées par les pouvoirs publics et inscrites dans la législation viennent initialement du secteur privé. Ces politiques et réglementations publiques, en association avec les normes relatives aux intrants, aux procédés et aux résultats, influencent considérablement le fonctionnement et le développement des marchés privés en termes de qualité de l'alimentation [Caswell et Mojduszka, 1996]. Du point de vue de la théorie économique, la distinction entre normes publiques et normes privées, en termes fonctionnels, dépend davantage des intérêts pris en classer des produits similaires et les décrivent grâce à une terminologie cohérente facilement compréhensible par les participants au marché. Les normes renvoient à un système de mesure, établi par la réglementation ou les autorités compétentes, et les catégories à un système de classification fondé sur des attributs quantifiables. 
compte lors de l'élaboration et de la mise en œuvre de la norme. Pour une norme publique, régie par le droit public et fixée par des instances gouvernementales, on suppose que les intérêts de tous les acteurs de l'économie - les producteurs, les consommateurs et la collectivité en général (externalités) - sont pris en considération. En revanche, les normes privées font intervenir les profits et les intérêts d'entreprises, de producteurs ou d'organismes privés. Elles peuvent en principe coïncider avec les intérêts des consommateurs, mais seulement dans la mesure où ceux-ci correspondent aux objectifs de l'entreprise et se reflètent dans le prix. Les normes peuvent également émaner d'organisations non gouvernementales $(\mathrm{ONG})$; elles appartiennent alors à une autre catégorie de normes privées. Les ONG, qui ont plutôt une vocation non lucrative, ne visent pas nécessairement les mêmes objectifs que les gouvernements en élaborant des normes [OMC, 2005]. Le tableau 1 propose une classification des différents types de normes publiques de qualités alimentaires, selon quatre dimensions de base : buts, caractéristiques, ampleur et étendue.

Tableau 1. Classification des normes alimentaires

\begin{tabular}{|l|l|}
\hline Dimension & Classification \\
\hline$\underline{\text { Buts }}$ & $\begin{array}{l}\text { Qualité }: \text { Règlements qui fournissent la } \\
\text { différentiation de produits lorsqu'ils sont bruts ou } \\
\text { lorsqu'ils sont transformés, non directement liés à } \\
\text { la santé et à la sécurité alimentaire }\end{array}$ \\
\hline Caractéristiques & $\begin{array}{l}\text { Caractéristiques de produit brut }: \text { Règlements qui } \\
\text { visent les aspects matériels de l'aliment. } \\
\text { Caractéristiques de transformation }: \text { règlements } \\
\text { qui visent à définir les étapes par lesquelles un } \\
\text { produit alimentaire est produit, traité ou distribué }\end{array}$ \\
\hline Ampleur & $\begin{array}{l}\text { Verticale }: \text { Règlements spécifiques à un produit } \\
\text { unique, voire étroitement lié, dans une ou plusieurs } \\
\text { étapes de la chaîne de commercialisation } \\
\text { Horizontale }: \text { Règlements appliqués entre des } \\
\text { produits non nécessairement très liés }\end{array}$ \\
\hline Étendue & $\begin{array}{l}\text { Uniforme }: \text { Règlements qui s'appliquent également } \\
\text { aux produits d'origine domestique et étrangère } \\
\text { Spécifique }: \text { Règlements qui s'appliquent } \\
\text { seulement aux produits importés, souvent } \\
\text { seulement de certaines origines }\end{array}$ \\
\hline
\end{tabular}

Source : d'après Josling, T., Roberts, D., et Orden, D., (2004), Food Regulation and Trade Towards a Safe and Open Global System, Institute of International Economics, Washington, DC, p. 18.

Les normes privées sont par définition volontaires, tandis que les normes publiques peuvent être volontaires ou obligatoires. Les normes volontaires consensuelles sont l'aboutissement de processus formels de concertation impliquant les différents acteurs du marché, avec ou sans la participation des pouvoirs publics [Henson et Reardon, 2005]. Les normes volontaires publiques sont souvent associées à des labels officiels, tels que ceux de l'UE : appellation d'origine protégée (AOP), indication géographique protégée (IGP) et spécialité traditionnelle garantie (STG). Ceux-ci ne renvoient généralement pas aux produits, bien qu'il existe aussi des normes de production (dans le domaine de la production biologique, par exemple). Les normes volontaires, publiques ou privées, assorties de labels attestant la conformité, sont un moyen de remédier au problème de l'asymétrie d'information. Les pays peuvent choisir entre différents types de procédés d'étiquetage qui allouent la tâche de fournir l'information aux secteurs public et privé de différentes manières. Ces différentes approches basées sur des règlements volontaires et obligatoires sont illustrées dans le tableau 2. Selon [Josling et al., 2004], le premier régime fournit un exemple d'une procédure d'étiquetage des produits alimentaires pour des indications géographiques. Ici l'industrie 
développe et adopte les normes qui permettent d'attribuer à un produit une région spécifique. Dans le deuxième régime le gouvernement joue un grand rôle dans le contrôle des revendications volontaires telles que celles sur l'identité du produit qui réduisent l'incertitude du consommateur et les possibilités de tromperie sur la qualité. Le troisième et quatrième régimes sont basés sur les procédures d'étiquetage qui nécessitent la divulgation obligatoire d'informations; dans un cas pour étiqueter un produit du pays de provenance et dans l'autre pour l'étiqueter selon ses caractéristiques physiques telle que la composition alimentaire.

En ce qui concerne les normes de qualité obligatoires (minimales), dans la mesure où seuls les produits qui répondent aux exigences sont autorisés à la vente, elles ont pour effet de réduire l'éventail des produits disponibles à la consommation, avec des implications potentielles sur les prix. Une définition large des normes inclut les règlements techniques obligatoires et les accords volontaires portant sur les caractéristiques qualitatives des biens et des services ${ }^{7}$. Les normes obligatoires sont établies par un organisme public de normalisation et ont un caractère contraignant. Bien que les normes privées ou propriétaires soient volontaires, dans bien des cas elles sont devenues de facto obligatoires, dès lors que la reconnaissance par les acteurs du marché, en raison d'une part de marché ou d'une pratique dominante, ou le respect de ces normes conditionne l'accès des fournisseurs à la chaîne de valeur de marques déposées de certaines grandes entreprises de distribution alimentaire ou de restauration.

Tableau 2. Rôles privés et publics dans l'étiquetage sur la qualité des aliments

\begin{tabular}{|c|c|c|c|c|}
\hline Rôle & Régime1 & Régime 2 & Régime 3 & Régime 4 \\
\hline Statut légal: & Volontaire & Volontaire & Obligatoire & Obligatoire \\
\hline Norme fixée par: & Secteur privé & Gouvernement & Gouvernement & Gouvernement \\
\hline Caractéristique: & Transformation & Brut & Transformation & Brut \\
\hline $\begin{array}{l}\text { Réalisation: } \\
\text { Évaluation de } \\
\text { conformité }\end{array}$ & $\begin{array}{l}\text { Adoption de } \\
\text { pratique de production }\end{array}$ & $\begin{array}{l}\text { Reformulation } \\
\text { produit }\end{array}$ & $\begin{array}{l}\text { Ségrégation et } \\
\text { Préservation de l'identité }\end{array}$ & $\begin{array}{l}\text { Reformulation } \\
\text { produit }\end{array}$ \\
\hline Accréditation: & Secteur privé & & & \\
\hline Certification: & Secteur privé & & & \\
\hline Évaluation: & & Secteur privé & & Secteur privé \\
\hline Documentation: & & & Privé & \\
\hline Inspection/audit: & & Gouvernement & Gouvernement & Gouvernement \\
\hline Exécution légale: & $\begin{array}{l}\text { Privée et } \\
\text { Gouvernement }\end{array}$ & Gouvernement & Gouvernement & Gouvernement \\
\hline Exemples: & $\begin{array}{l}\text { Indications } \\
\text { Géographiques }\end{array}$ & $\begin{array}{l}\text { Identités des } \\
\text { normes }\end{array}$ & $\begin{array}{l}\text { Pays d'origine } \\
\text { Des étiquettes }\end{array}$ & $\begin{array}{l}\text { Étiquette } \\
\text { contenu } \\
\text { alimentaire }\end{array}$ \\
\hline
\end{tabular}

Source : d'après Josling, T., Roberts, D., et Orden, D., (2004), Food Regulation and Trade, op. cit. ,p. 131.

7 L'Accord relatif aux obstacles techniques au commerce (OTC) de l'OMC, pour sa part, établit une distinction nette entre les règlements techniques obligatoires et les normes volontaires. L'expression «norme de facto obligatoire » est utilisée dans le sens où la norme privée d'une société d'achat de produits d'alimentation devient pratiquement obligatoire pour les sociétés d'approvisionnement qui souhaitent être capables de fournir ou vendre des produits alimentaires à la société d'achat en aval. Cela diffère de l'utilisation du terme dans l'économie de l'innovation. Dans les ouvrages considérés, le terme « normes de facto » concerne les normes techniques (formelles ou informelles) qui l'emportent en pratique, soit parce qu'elles ont été imposées, soit parce qu'elles occupent une position de force sur le marché. Cela contraste avec « les normes de jure » exigées conformément à la loi et établies selon un règlement ou peut-être au moyen d'une norme volontaire approuvée par une organisation de normes comme ISO (l'organisme international de normalisation) en réponse à un besoin largement perçu. 


\section{Encadré 2. Paradigme des normes}

Le tableau 2 met en évidence les différents types de normes. Henson [2004] conçoit les normes comme un continuum variant en fonction du degré de liberté des utilisateurs, c'est-à-dire de la marge de manœuvre dont ils disposent en termes d'obligation de mise en conformité (figure 1). À l'une des extrêmités se situent les réglementations publiques contraignantes définissant des normes ou des catégories permettant de garantir une qualité et une sécurité minimales ; à l'autre extrêmité, des normes volontaires que les utilisateurs peuvent choisir ou non d'appliquer, choix dont ils assument toutes les conséquences économiques. Les normes volontaires peuvent devenir de facto obligatoires ou obligatoires sur participants au marché, dans la mesure où les fournisseurs sont contraints de les respecter pour entrer ou rester sur un marché donné. Ainsi, certaines normes propriétaires volontaires établies par les acheteurs de la filière alimentaire peuvent exclure du marché des fournisseurs potentiels d'aliments qui ne remplissent pas les conditions requises.

Encadré 2. Tableau 1. Comparaison des normes

\begin{tabular}{|c|c|c|c|c|}
\hline Type de norme & Origine & Fonction & Conformité & Contrôle/vérification \\
\hline \multirow[t]{2}{*}{$\begin{array}{l}\text { Réglementation } \\
\text { publique }\end{array}$} & $\begin{array}{l}\text { Mise en œuvre par } \\
\text { l'État }\end{array}$ & \multirow{2}{*}{$\begin{array}{l}\text { Protection du } \\
\text { consommateur, } \\
\text { prévention des } \\
\text { fraudes }\end{array}$} & Obligatoire & \multirow[t]{2}{*}{$\begin{array}{l}\text { Certification et inspections } \\
\text { officielles }\end{array}$} \\
\hline & & & & \\
\hline \multirow{3}{*}{$\begin{array}{l}\text { Norme } \\
\text { publique/privée }\end{array}$} & \multirow{3}{*}{$\begin{array}{l}\text { Résultat d'un } \\
\text { consensus entre } \\
\text { représentants } \\
\text { publics et privés du } \\
\text { secteur }\end{array}$} & \multirow{2}{*}{$\begin{array}{l}\text { Mécanisme } \\
\text { général du type } \\
\text { HACCP }\end{array}$} & Volontaire & \multirow{3}{*}{$\begin{array}{l}\text { Conformité certifiée par } \\
\text { première, seconde ou tierce } \\
\text { partie }\end{array}$} \\
\hline & & & & \\
\hline & & & & \\
\hline \multirow[t]{3}{*}{ Norme privée } & \multirow{3}{*}{$\begin{array}{l}\text { Créée par une } \\
\text { entreprise, à usage } \\
\text { interne, ou par un } \\
\text { groupe de } \\
\text { producteurs et } \\
\text { reconnue par l'État }\end{array}$} & \multirow{3}{*}{$\begin{array}{l}\text { Norme élaborée } \\
\text { en fonction des } \\
\text { dispositions } \\
\text { publiques en } \\
\text { vigueur ou d'un } \\
\text { produit particulier } \\
\text { et du procédé de } \\
\text { production } \\
\text { correspondant }\end{array}$} & Volontaire & \multirow{3}{*}{$\begin{array}{l}\text { Conformité garantie par des } \\
\text { contrôles internes ou certifiée } \\
\text { par un organisme } \\
\text { indépendant ou un service } \\
\text { public }\end{array}$} \\
\hline & & & & \\
\hline & & & & \\
\hline \multirow[t]{4}{*}{$\begin{array}{l}\text { Norme privée } \\
\text { propriétaire }\end{array}$} & \multirow{4}{*}{$\begin{array}{l}\text { Créée par une } \\
\text { entreprise de } \\
\text { distribution, de } \\
\text { restauration ou de } \\
\text { production } \\
\text { alimentaire pour } \\
\text { coordonner et } \\
\text { maîtriser les } \\
\text { approvisionnements }\end{array}$} & \multirow{4}{*}{$\begin{array}{l}\text { Régit les } \\
\text { relations entre un } \\
\text { acheteur et des } \\
\text { fournisseurs } \\
\text { privés }\end{array}$} & $\begin{array}{l}\text { Volontaire mais } \\
\text { entrant }\end{array}$ & \multirow{4}{*}{$\begin{array}{l}\text { Conformité assurée par un } \\
\text { audit de seconde partie ou } \\
\text { par la certification d'un } \\
\text { organisme indépendant }\end{array}$} \\
\hline & & & $\begin{array}{l}\text { generalement } \\
\text { dans des }\end{array}$ & \\
\hline & & & $\begin{array}{l}\text { relations } \\
\text { contractuelles }\end{array}$ & \\
\hline & & & & \\
\hline
\end{tabular}

Source : Valceschini, E., et Saulis, L. (2005), Articulation entre réglementation, normalisation et référentiels privés dans les industries agroalimentaires. Rapport final, rapport d'une étude financée par le ministère de l'Agriculture et de la Pêche - Direction des Politiques Économique et Internationale (référence MAP 05 D1 05 01). 


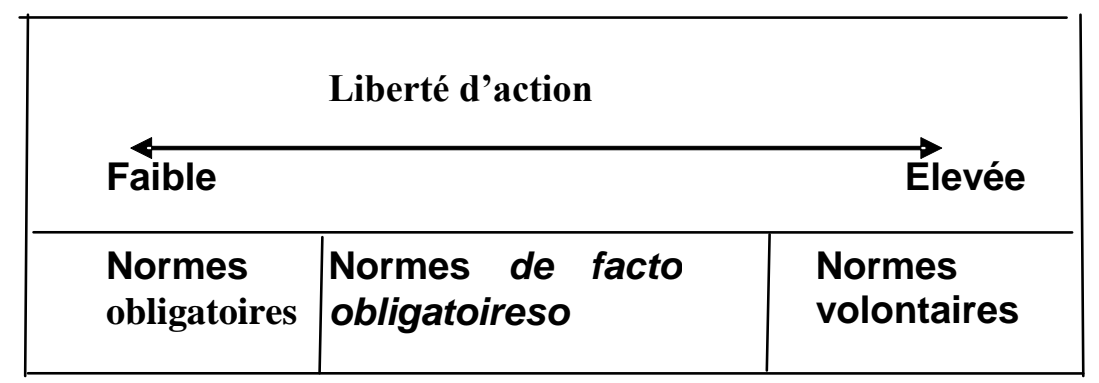

Source : S. Henson et J. Caswell [1999].

La distinction entre normes obligatoires et volontaires permet de définir une classification bidimensionnelle (figure 2) largement utilisée dans les publications spécialisées [Henson, 2005]. Cette classification peut servir à comparer la normalisation des produits et des procédés à l'intérieur d'un pays et d'un pays à l'autre. Par exemple, dans le cas de pays peu développés, la plupart des normes s'inscrivent dans le quadrant « obligatoire/publique » de la figure 2. Bien que des normes publiques puissent être prévues dans les règlements des pays les moins avancés, elles n'ont pas nécessairement la portée réelle voulue si elles ne sont pas mises à exécution par les autorités, faute de ressources. À mesure que l'économie se développe et s'intègre au marché mondial, l'incidence des normes publiques volontaires progresse, notamment à l'exportation, et les normes privées se multiplient en conséquence. Dans les pays développés, de nombreuses normes privées propriétaires sont devenues de facto obligatoires pour les acteurs du marché, dans la mesure où les entreprises dominantes imposent leurs exigences aux fournisseurs par le biais d'une concurrence commerciale fondée sur la différenciation qualitative.

Sur la plupart des marchés, normes volontaires et normes obligatoires coexistent, moyennant d'innombrables interactions et liens d'interdépendance. Dans le passé, la plupart des normes instaurées par des instances réglementaires publiques avaient un caractère obligatoire, tandis que celles appliquées dans le privé étaient volontaires. Toutefois, des normes volontaires ont été progressivement mises au point par des organismes publics (cas du Label rouge en France), tandis que les normes d'aliments des grands distributeurs ou compagnies de restauration en position de force sur les marchés deviennent parfois obligatoires de facto. Les normes volontaires peuvent aussi prendre la forme d'un mécanisme qui facilite le respect des normes obligatoires ou apporte des preuves de conformité. Par exemple, le respect de normes volontaires (comme les systèmes HACCP - Analyse des risques points critiques pour leur maîtrise) peut servir à prouver qu'une entreprise observe la " diligence requise "vis-à-vis des normes légales de fiabilité d'un produit. Les normes obligatoires peuvent aussi faire entrer des normes privées dans leurs exigences. Elles suscitent alors une utilisation volontaire et une adhésion plus grandes. Les organismes de normalisation sont aussi amenés à consulter les entreprises durant le processus d'élaboration des normes ; lorsque les normes volontaires se généralisent, elles peuvent être incorporées aux normes publiques obligatoires. Ainsi, normes volontaires et normes obligatoires peuvent être considérées comme complémentaires et d'égale importance dans le système de normalisation.

Une autre distinction peut être établie entre les normes nationales et celles qui sont élaborées dans un cadre commun ou multilatéral. Dans certains cas, l'adoption de normes internationales peut être une façon rentable d'améliorer les normes nationales pour les adapter aux exigences des marchés mondiaux. L'efficacité de cette approche dépend du degré d'adéquation entre les normes internationales et les besoins et la situation du marché national. Si les conditions locales ne sont pas suffisamment prises en compte, les coûts de mise en conformité peuvent dépasser ceux des normes nationales. 


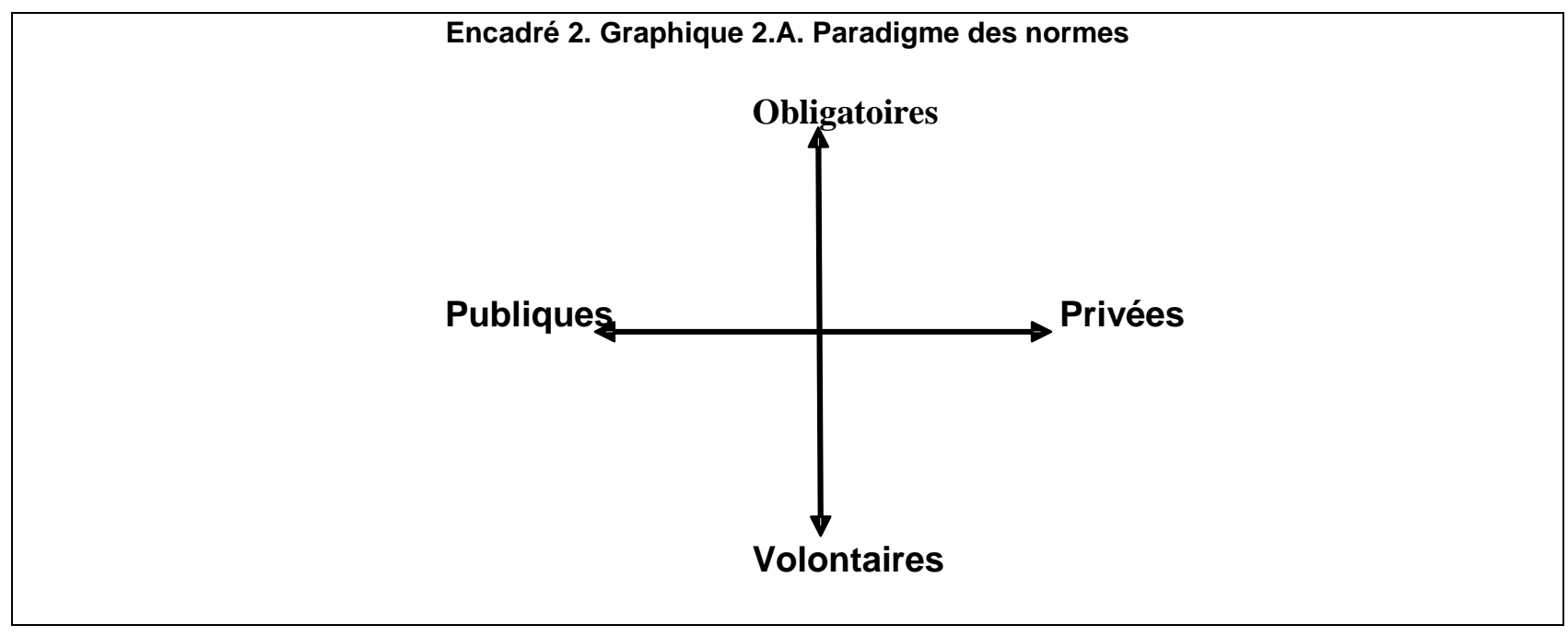

Source : Henson, S. (2007).

La plupart des normes, à commencer par les règlements obligatoires, ont pour effet d'accroître les coûts de production et de mise en conformité, du moins pour certaines entreprises de la chaîne alimentaire. Les normes de produits peuvent avoir moins d'incidences sur les coûts que les normes de procédés, puisqu'elles laissent davantage de latitude pour choisir des méthodes de production moins coûteuses [Unnevehr et Jensen, 1996; Segerson, 1998]. La mise en œuvre des normes induit normalement une augmentation des coûts pour les consommateurs, qui devront acquitter un prix plus élevé, et des choix plus limités. Alors que l'évolution des normes (privées) reflète en partie celle de la demande et des préférences des consommateurs, la consommation alimentaire est de plus en plus influencée par les normes. Les normes publiques et privées tendent en effet à proliférer et à se diversifier. Au renforcement des exigences réglementaires, accompagnant les progrès scientifiques et techniques et l'approfondissement des connaissances, s'ajoute l'aspiration grandissante des consommateurs à des produits plus divers et de meilleure qualité. L'« escalade » générale des normes de qualité se traduit en outre par une coordination plus étroite des normes publiques et privées. Reste à savoir si le système de normes alimentaires va y gagner globalement en cohérence. Par ailleurs, étant donné l'importance croissante dans le secteur de l'alimentation de normes volontaires privées qui supposent bien souvent une amélioration en termes de qualité, quel rôle doivent jouer les normes publiques de qualité alimentaire dans un secteur dynamique ?

\section{La raison d'être des normes alimentaires publiques}

Compte tenu de la diversité des obligations informatives prévues, la classification des biens en fonction des informations dispensées aux consommateurs qui vient d'être examinée dans la section précédente pose des problèmes différents pour la normalisation et à la réglementation publiques. Dans le cas des biens de recherche, étant donné que les producteurs courent le risque d'une baisse de leur activité et de leur chiffre d'affaires s'ils vendent des biens de qualité inférieure, ils ont tout intérêt à proposer aux consommateurs l'éventail qualitatif pour lequel ceux-ci sont prêts à payer. Pour ce type de produits, les consommateurs disposent d'une information qualitative quasi-parfaite avant de les avoir achetés. Ils sont donc généralement capables de se protéger, ou du moins n'acceptent que temporairement de mettre la main au porte-monnaie, mais pas de risquer leur santé, si un produit ne satisfait pas à leurs attentes. En conséquence, les marchés de biens et de caractéristiques de recherche ne seront généralement qu'une préoccupation mineure pour les responsables de la réglementation.

Sur les marchés des biens d'expérience, en revanche, qui répondent à de nombreux autres aspects de la qualité des aliments, les producteurs sont mieux informés que les consommateurs sur la qualité des 
produits proposés à la vente. Comme les consommateurs ne peuvent identifier les caractéristiques qualitatives des biens d'expérience qu'après consommation, la réglementation publique a plus de chances d'être utile ici que dans le cas des biens de recherche, que ce soit en exigeant un étiquetage informatif ou par le biais d'une loi sur la responsabilité du fait des produits. Les réglementations sur l'étiquetage pallient en partie les imperfections du marché résultant d'un manque d'information et ont pour effet de transformer les caractéristiques d'expérience des biens en caractéristiques de recherche. Ces marchés peuvent s'autocorriger, puisque les consommateurs finissent par disposer d'une information complète du fait peut-être d'achats répétés du même produit alimentaire, et il n'existe donc aucune asymétrie d'information, ni aucun autre facteur de défaillance du marché à long terme. Il ressort des publications spécialisées que dans cette situation, l'intervention autoritaire d'organismes publics provoque un recul du bien-être social [Bocksteal, 1984].

Le déficit d'information sur la qualité des aliments dont souffrent les consommateurs est même plus grave dans le cas des caractéristiques de confiance, raison pour laquelle les décideurs publics choisissent souvent d'intervenir en imposant une norme obligatoire ou un label de qualité qui permettra de différencier les produits concernés. La surveillance réglementaire des revendications de qualité des producteurs est vue comme une responsabilité du secteur public, particulièrement pour les nombreux produits alimentaires qui ne portent aucune marque et où les mécanismes qui permettent d'associer une entreprise agroalimentaire particulière avec un produit sont manquants et ne permettent donc pas de protéger l'intégrité de transactions du marché. Ce type de réglementation peut résoudre le problème de l'information, remédier à la défaillance du marché et accroître l'efficience de celui-ci. La réglementation publique directe peut prendre la forme de normes sur les procédés de fabrication ou d'une spécification du niveau de qualité requis pour le produit final. Les normes constituent donc un mécanisme permettant aux autorités publiques de réguler le système alimentaire de manière à garantir le niveau de qualitésouhaité par la collectivité. Les normes obligatoires agissent alors comme des normes minimales, en excluant du marché les produits ne répondant pas aux critères de qualité souhaités requis [OMC, 2005]. Les normes publiques améliorent ainsi la communication des informations sur la qualité des aliments commercialisés et présentent l'avantage de permettre une estimation claire et équilibrée des divers arbitrages en jeu. En décourageant les activités irresponsables et récompensant les initiatives allant dans le bon sens, ces normes peuvent inciter les industries agroalimentaires à davantage de transparence. Il est en outre plus facile aux instances réglementaires qu'aux acteurs du secteur privé d'instaurer avec certitude une norme unique - une norme «biologique, par exemple, d'autant qu'elles peuvent poursuivre toute personne enfreignant la réglementation en vigueur [McCluskey, 2006]. Il ne faut toutefois pas oublier que les pouvoirs publics peuvent être soumis à des pressions de groupes d'intérêt et que si les normes qu'ils mettent en place sont obligatoires, elles seront davantage susceptibles de servir de mécanisme d'exclusion. Dès lors que des intérêts particuliers seraient pénalisés par une modification réglementaire, ils pourraient avoir intérêt à bloquer le processus, ce que pourraient faciliter les procédures officielles d'élaboration des normes dans certains pays. Bien qu'il soit difficile de généraliser, les normes publiques peuvent être moins souples que les protocoles privés pour réagir aux changements de circonstances et ne sont généralement pas particulièrement propices à l'innovation. Une fois adoptées, les normes publiques pourront difficilement être modifiées pour s'adapter aux évolutions scientifiques et technologiques ou à celles des préférences des consommateurs. De plus, le coût de leur élaboration sera supporté par les contribuables et non par leurs bénéficiaires potentiels, d'où un risque d'allongement et de renchérissement du processus. Aux Etats-Unis, par exemple, l'adoption de la norme «biologique » a nécessité quelque huit années [Ippolito, 2003]. Cependant, ce problème de norme enfermée dans une voie qui pourrait ne pas être optimale est aussi un problème apparent pour quelques normes industrielles qui ont été efficacement imposées par une ou deux sociétés. Des exemples peuvent être trouvés dans des secteurs où les normes de compatibilité sont cruciales, et où il existe des avantages pour celui qui prend les premières parts de marchés car il peut avoir la priorité sur la qualité de produit (la norme DVD actuelle par exemple). 
Les normes publiques revêtent souvent la forme de normes minimales de qualité obligatoires (voir encadré 1) et ont pour effet d'accroître la présence d'une caractéristique souhaitée dans les produits alimentaires en question. Le contrôle de leur application passe traditionnellement par l'inspection officielle des locaux de production et/ou des produits finals, opération dont le coût pour l'Etat est considérable [Henson, 2006]. L'existence de normes publiques de qualité des aliments ayant force de loi, qui a joué un rôle important dans l'évolution des marchés alimentaires, peut avoir une influence non négligeable sur leur fonctionnement même. Dans la plupart des cas, les normes publiques ont eu pour corollaire une amélioration de la qualité des aliments. C'est ainsi que les taux minimum de matière grasse et de matière sèche auxquels doit satisfaire le lait liquide en augmentent la valeur nutritionnelle. Par ailleurs, les réglementations sur la qualité des produits, telles que les normes de classement des fruits frais, permettent d'obtenir certaines caractéristiques souhaitables au niveau de la consommation ainsi que divers aspects de bien public. A titre d'exemple, une réglementation interdisant ou limitant les résidus de pesticides sur les fruits et légumes aura vraisemblablement pour effet de diminuer les quantités de pesticides appliquées par les agriculteurs et contribuera ce faisant à améliorer la qualité de l'environnement [Sunding, 2003]. Ces répercussions positives sur l'amélioration de la qualité ne sont cependant pas systématiques. Prenons le cas bien connu du tout premier système de classement de la viande mis en place au Etats-Unis. En effet, comme les normes instaurées ne définissaient pas de manière suffisamment précise les caractéristiques de qualité de la viande de bœuf destinée à la consommation, elles servaient davantage à compenser l'accroissement du cheptel (autrement dit, les quantités de viande produites) qu'à améliorer la qualité de la viande, ce qui a entraîné un recul de la consommation intérieure à mesure que la qualité baissait. Face à ces carences sont alors apparues des conventions privées telles que les marques ou les labels de qualité, l'USDA se transformant ainsi en auditeur indépendant chargé de vérifier de la bonne application de ces normes privées [Ferrier et Lamb, 2007].

La finalité première des normes alimentaires publiques est de réduire les risques pour la santé humaine dus à la consommation d'aliments de qualité inférieure ou en dessous du standard de qualité et de protéger les consommateurs de la fraude et des pratiques abusives des distributeurs sur la teneur et la qualité des produits s'inscrivant dans une démarche volontaire [Henson et Traill, 1993 ; Antle, 1995]. Le souci de protéger les consommateurs et de promouvoir certaines valeurs (les productions locales, par exemple) et le processus de préparation alimentaires a conduit les pouvoirs publics à intervenir également dans le contrôle des aspects non sanitaires des caractéristiques qualitatives des aliments (pays d'origine, label biologique, appellation d'origine protégée ou AOP, indication géographique protégée ou IGP, OGM, etc.) [Garcia Martinez et al., 2007]. Catégories et normes existent depuis longtemps sur les marchés céréaliers, où elles ont d'abord été introduites pour faciliter les transactions de pleine concurrence portant sur des biens largement homogènes ${ }^{8}$. Elles ont aussi permis d'empêcher les usages abusifs ou frauduleux relatifs aux poids et mesures, par exemple dans le cas des pierres et autres corps étrangers ajoutés à la farine pour en augmenter le poids lors des transactions entre minoteries et acheteurs [Kindlberger, 1983 ; Hill, 1990]. Dans le contexte des marchés de masse de produits de base très homogènes, les normes publiques présentent des caractéristiques de biens publics, permettent d'abaisser les coûts de transaction grâce aux économies d'échelle réalisées, réduisent les coûts de recherche supportés par les intervenants sur les marchés, et continueront probablement de prévaloir sur les normes privées [Banque mondiale, 2005]. La mondialisation des systèmes agroalimentaires et l'abandon des marchés de masse au profit de marchés de produits fortement différenciés font cependant évoluer le rôle joué par les normes et classes. Celles-ci ne vont plus tant porter sur les résultats (caractéristiques mesurables des produits) que sur les procédés (par exemple : modes de fabrication, de transformation, de distribution, etc.) [Clayton et al., 2003].

Les termes «norme» et «classe » se recouvrent en partie. Ils se distinguent principalement par le fait qu'une classe place un produit dans une catégorie qualitative donnée, tandis qu'une norme représente un seuil mesurable que doit respecter un produit alimentaire pour pouvoir prétendre à une appellation particulière (norme d'identification) ou à une classe particulière (norme de classement) [Gardner, 2003]. 
Les instances réglementaires peuvent également aider à pallier les défaillances du marché dues aux incertitudes sur la qualité en promulguant des normes de définition qui contribueront à empêcher la fraude et la tromperie sur la qualité des produits offerts à la consommation [Becker, 1999]. Ces normes peuvent s'avérer utiles s'agissant de la qualité des procédés et des caractéristiques spécifiques au processus de production, par exemple pour la définition de la production biologique. C'est, semble-t-il, ce qui s'est passé aux États-Unis, où l'USDA a fixé une norme biologique nationale donnant une définition de ce qu'est un produit biologique. Avant la promulgation de cette norme, certains aliments biologiques bénéficiaient d'une certification délivrée dans le cadre de programmes mis en place par les États ou le secteur privé, mais il arrivait souvent que les consommateurs ne sachent pas exactement ce que recouvrait ce terme de biologique, d'où les risques de fraude et de présentation trompeuse de la qualité des produits. Dans divers pays européens, des normes publiques volontaires sont fréquemment combinées à des labels publics, comme les AOP, les IGP ou les spécialités traditionnelles garanties (STG), qui ont été instaurées au bénéfice des producteurs agricoles privés. Dans ces différents cas de figure, la garantie de qualité est le résultat d'une coopération entre secteur public et secteur privé. Les producteurs privés et l'instance collective qui les représente fixent les détails du procédé de production décrit par la norme. Les critères d'attribution de ces labels officiels imposent que l'inspection, le contrôle et la certification soient réalisés par une tierce partie indépendante normalement accrédités par l'instance publique de réglementation, afin de garantir la présence des caractéristiques de confiance, attestée en se fondant sur des procédés traditionnels de production et une origine spécifiée [Young et Hobbs, 2002].

Pour certains aspects qualitatifs, liés à la santé humaine, on ne sait toutefois pas très bien quel est le degré d'acceptabilité de l'étiquetage informatif pour les autorités publiques, les entreprises agroalimentaires et les consommateurs [Caswell et Mojduszka, 1996]. En ce qui concerne les pouvoirs publics, ils préférer généralement éviter tout risque (autre peut être que l'aléa moral) en promulguant une norme obligatoire. Ces dernières années, les normes publiques se sont durcies et ont gagné en complexité ; l'accent a davantage été mis sur les aspects scientifiques et une approche systémique. Parallèlement, dans de nombreux pays, les pouvoirs publics ont cherché à réduire le poids de la réglementation pour les industries agroalimentaires. Certaines mesures fondées sur des obligations de résultats ont été adoptées, qui permettent aux fournisseurs d'atteindre la qualité souhaitée de façon plus souple et plus rentable [Flynn et al., 2003]. Pour le contrôle des procédés de fabrication, prenant acte de l'inefficacité et du caractère onéreux des contrôles de qualité des produits finaux, on a progressivement introduit des critères basés sur les principes de l'analyse des risques-points critiques pour leur maîtrise (HACCP). Les modalités d'application de ces réglementations se répercutent sur le niveau et le mode de mise en œuvre adoptés par les pouvoirs publics pour garantir la qualité des aliments. Les règlements fondés sur ldes obligations de résultats, lorsqu'ils sont associés à des normes HACCP et à une traçabilité des produits, reposent principalement sur le contrôle des audits d'entreprise portant sur les informations relatives à la production. Ces dispositifs remplacent les visites régulières d'inspection des produits et des locaux prévues par les procédures traditionnelles, ce qui réduit les coûts du contrôle et confère à l'État un rôle de surveillance du système de qualité des aliments et de ses résultats.

Étant donné le glissement qui s'opère dans un certain nombre de pays entre une réglementation des produits et une réglementation des procédés ou l'adoption de critères de résultats à des fins de qualité alimentaire (critères HACCP, par exemple), une des questions qui se posent est de savoir si la mise en conformité doit être obtenue au moyen de normes volontaires ou de normes obligatoires. L'argument traditionnellement mis en avant en faveur des dispositifs volontaires est qu'ils sont plus avantageux pour les entreprises, car ils entraînent des coûts de mise en conformité et de transaction inférieurs à ceux résultant des normes obligatoires. Néanmoins, dans tous les pays développés, apparaissent des procédures réglementaires basées sur le système HACCP et rendues obligatoires pour certains aliments et produits transformés. Cette évolution amène à se demander si l'intervention autoritaire des pouvoirs publics implique nécessairement des coûts de mise en conformité et de transaction plus élevés. Si ce n'est pas le cas, la menace représentée par les réglementations contraignantes peut ne pas constituer pour les 
entreprises une incitation suffisante à adopter des mesures volontaires pour améliorer la qualitéde leurs produits, et les approches obligatoires peuvent accrôtre le bien-être [Venturini, 2002]. C'est peut-être ce qui explique que beaucoup de pays développés se tournent de plus en plus vers des systèmes obligatoires fondés sur les critères HACCP.

Signe de l'évolution du rôle joué par le secteur public, certains pays transfèrent au secteur privé de plus en plus de responsabilités en matière de qualité alimentaire, soit sous la forme de protocoles privés, soit sous celle de certifications de conformité délivrées par des tiers. À titre d'exemple, on peut citer les procédures de contrôle mises en place en France pour les importateurs de fruits et légumes. Depuis 1998, il incombe en effet aux importateurs de vérifier la qualité de leurs produits dans le cadre d'un système d'autocontrôle visant à s'assurer que les limites maximales de résidus de pesticides et d'autres produits chimiques présents sur les fruits et légumes respectent la norme française. Une convention d'autocontrôle établie sur la base des procédures HACCP est négociée entre les différents acteurs de la distribution et l'instance collective les représentant, puis approuvée par les autorités publiques. Cette instance est chargée de veiller à ce que ses adhérents réalisent les contrôles requis et respectent leurs engagements. La menace d'exclusion suffit à garantir la bonne application de la convention. Les autorités publiques, qui procédaient jusqu'alors aux inspections, se contentent désormais de vérifier que les distributeurs effectuent correctement les autocontrôles, ce qu'elles font en examinant les rapports d'autocontrôle soumis par ces derniers. Cette délégation des analyses qualitatives aux distributeurs eux-mêmes répond à un certain nombre d'objectifs, notamment : accroître le nombre et le champ d'application des contrôles qualité opérés sur le marché et améliorer leur efficacité, réduire le coût des inspections effectuées par les services publics et dégager les ressources correspondantes pour les affecter à d'autres secteurs où les risques peuvent être supérieurs (produits à base de viande, par exemple), et axer davantage les contrôles officiels sur les résultats. Cette délégation de responsabilités permet aux distributeurs de réduire l'ampleur et la fréquence des contrôles officiels et de partager avec d'autres le coût du processus d'autocontrôle, puisque toute analyse scientifique requise est confiée à un même laboratoire qui leur accorde un tarif préférentiel, d'où des économies d'échelle dont ils bénéficient. Enfin, en cas de problèmes de qualité, cette délégation diminue aussi l'importance de leur responsabilité juridique dès lors que les autorités publiques considèrent qu'ils ont mobilisé des ressources suffisantes aux procédures d'autocontrôle. A partir du moment où les autocontrôles sont correctement effectués, les distributeurs ne peuvent en aucun cas être responsables pénalement pour non-conformité. Dans une telle hypothèse, le distributeur doit notifier l'infraction aux normes de qualité décelée à son instance représentative, qui en informe les autorités publiques [Codron et al. 2005].

L'exemple décrit ci-dessus illustre bien le développement en cours, dans certains pays, d'une coordination et d'une coopération entre les acteurs des secteurs public et privé dans le domaine des normes alimentaires. Il est également intéressant d'évoquer l'exemple de l'évolution de la réglementation de l'UE sur la qualité des aliments. Depuis l'entrée en application, le 1er janvier 2006, des divers règlements européens sur l'hygiène alimentaire, il incombe aux professionnels de la filière agroalimentaire de produire des aliments sans risque pour la santé humaine, toutes les entreprises étant tenues de procéder à des contrôles prouvant qu'elles ont intégré des mesures de qualité alimentaire dans leur gestion. Autrement dit, l'approche de contrôle largement prescriptif a été abandonnée au profit d'une démarche d'autoréglementation, les instances réglementaires imposant aux entreprises agroalimentaires de définir et de mettre en œuvre leurs propres règles et procédures internes pour atteindre les objectifs qui leur ont été fixés. Il appartient ensuite aux autorités réglementaires d'approuver ces règles internalisées et d'en contrôler la bonne application. Les États membres de l'UE ne sont pas tenus d'aller au-delà de la transposition de la réglementation européenne dans leur droit national, mais il existe bien souvent des domaines dans lesquels il leur est permis d'adopter des dispositions supplémentaires spécifiques [Garcia Martinez et al., 2007]. On observe également une évolution de la réglementation aux États-Unis, où un processus à caractère essentiellement prescriptif et des normes de produit laissent la place à des normes plus souples, reposant sur une obligation de résultats, qui accordent aux entreprises davantage de latitude 
pour le choix des modalités de mise en œuvre. Au Canada, par contre, l'approche prédominante est basée principalement sur des normes de produit et de procédé détaillées qui reste prédominante, le respect de leur application étant contrôlé par le biais d'inspections sur site. Dans le secteur de la transformation de la viande et de la volaille, où les réglementations sur la qualité des aliments s'orientent désormais vers un régime de responsabilité des entreprises s'accompagnant d'une application obligatoire des procédures HACCP, les autorités ont accordé aux transformateurs une certaine flexibilité pour la mise en œuvre de systèmes de contrôle répondant à leurs besoins particuliers [Garcia Martinez et al., 2007]. Les services officiels d'inspection procèdent ensuite à l'audit des procédures de contrôle et des comptes rendus de résultats. On ignore cependant si le passage d'une approche à caractère prescriptif à une démarche d'autoréglementation observé dans un certain nombre de pays convient pleinement à l'ensemble des acteurs concernés, et en particulier aux consommateurs.

D'autres formes de coréglementation peuvent être envisagées, dans lesquelles les organismes publics peuvent davantage faire appel à des mécanismes privés de contrôle de la qualité des aliments, notamment la mise en œuvre de systèmes tels que la série ISO 22000. Le respect de ces normes de type système peut, par exemple, permettre aux autorités de contrôle d'établir une distinction entre produits et établissements alimentaires à risque faible ou élevé et de programmer leur calendrier d'inspection en conséquence. Pour ce faire, les autorités doivent toutefois être convaincues que les normes privées peuvent effectivement permettre d'évaluer le respect des normes publiques légales et d'inciter les entreprises à poursuivre en ce sens. En toute logique, la coordination de la gestion publique et privée de la qualité des aliments devrait permettre d'en améliorer le niveau à moindre coût et avec une allocation plus efficace les ressources réglementaires. Néanmoins, le champ de la coréglementation peut dépendre de la nature des dispositifs institutionnels et réglementaires en place, lesquels varient d'un pays à l'autre. La récente refonte de la réglementation européenne dans ce domaine semble offrir à cet égard un large éventail de possibilités de rapprochement entre les instances réglementaires et le secteur privé, mais en pratique, la coréglementation reste peu développée. Pour ce type de procédures, la formulation de mesures coordonnées de qualité alimentaires poursuivant des objectifs à la fois privés et sociaux demeure un véritable défi [Garcia Martinez, 2007]. D'autres formes de coordination entre secteur public et secteur privé existent depuis de nombreuses années: en témoignent les labels de qualité reposant sur les indications géographiques protégées (IGP), les appellations d'origine protégée (AOP) et les spécialités traditionnelles garanties (STG), qui ont été mises en place au profit des producteurs agricoles en France et dans d'autres parties de l'Europe et font désormais partie intégrante du droit de l'Union européenne. Comme cela a été noté, ces normes sont mises au point par les producteurs privés et une instance collective les représentant, les autorités publiques veillant à leur bonne application par le biais d'une certification.

Dans certains cas, cette transition vers une responsabilité accrue du secteur privé est renforcée par une réglementation sur la responsabilité ex post du fait des produits, dont l'objectif est avant tout d'inciter efficacement les producteurs à vendre des produits d'un niveau de qualité acceptable. Outre qu'elles respectent des normes de produits et de procédés plus strictes, les entreprises sont donc également responsables juridiquement des dommages ou préjudices causés par leurs produits. L'existence de cette réglementation montre bien la place prépondérante acquise, d'une part, par les normes de responsabilité délictuelle [Buzby et al., 2001] et, d'autre part, par l'obligation de prudence incombant aux distributeurs, qui doivent s'acquitter de leurs obligations selon le principe de la «diligence raisonnable » [Henson et Northen, 1998 ; OCDE, 2004 ; Henson, 2006]. C'est pour cette raison que l'introduction d'une exception de «diligence raisonnable» dans le cadre d'une législation ordonnée au Royaume-Uni a fortement dynamisé la mise en place de normes privées plus rigoureuses [Hobbs et Kerr, 1992].

Cette disposition d'exception impose aux distributeurs d'anticiper en s'assurant que les produits alimentaires qu'ils manipulent et tout produit alimentaire obtenu auprès de fournisseurs amont satisfont aux normes alimentaires en vigueur. Initialement, c'est aux détaillants qu'il appartenait de répondre à cette obligation en renforçant les contrôles de leurs produits et de ceux de leurs fournisseurs, lesquels étaient 
réalisés dans le cadre d'un audit externe. Ces dispositions ont été complétées par la mise en place, dans l'UE, d'un système d'alerte rapide s'appuyant sur un dispositif de traçabilité des produits, qui permet de repérer, grâce à une base de données publique, les produits non conformes aux réglementations en vigueur [Henson, 2006]. L'instauration d'une réglementation sur la responsabilité du fait des produits a encouragé les entreprises à adopter des normes plus exigeantes que les normes publiques et les procédures officielles d'autorisation [Antle, 1995]. La mise en jeu éventuelle de la responsabilité des fournisseurs peut donc assurer la conformité avec le règlement de qualité alimentaire public. Parallèlement, le rôle du secteur public a évolué, puisqu'il assume désormais des fonctions d'audit ou de surveillance des résultats obtenus par les entreprises privées appliquant un système de contrôle, ainsi que des fonctions de suivi basé sur des obligations de résultats [Jaffee et Henson, 2004].

Les divergences fondamentales qui existent entre les différents systèmes et institutions juridiques créés par les pays pour protéger les consommateurs contre la pauvre qualité des aliments expliquent en partie les divergences de conception du rôle des pouvoirs publics pour établir et garantir la conformité avec les réglementations alimentaires publiques. La différence de traitement juridique entre les réglementations a priori et les arbitrages a posteriori aide à comprendre les disparités qui existent entre les normes publiques des différents pays [Buzby et Frenzen, 1999]. Cette dernière approche n'étant pas encore très répandue, les mesures réglementaires contraignantes imposant la mise en œuvre a priori de normes obligatoires pour assurer l'approvisionnement en nourriture qui répond à des exigences de qualité.

\section{E. L'essor des normes et protocoles privés}

Pour l'essentiel, les publications traitant des normes ont jusqu'ici concerné le rôle des normes publiques sur la qualité des aliments à la fois comme instruments d'action et comme barrières potentielles aux échanges [Henson et Hooker, 2001]. Alors que les normes publiques sont depuis de nombreuses années présentes dans les systèmes alimentaires nationaux, les normes privées ne sont apparues qu'assez récemment dans le paysage de la qualité alimentaire, leurs objectifs et champ d'application étant extrêmement variables d'un pays et d'un produit à l'autre. Les normes privées se sont multipliées ces dernières années dans un certain nombre de pays industrialisés, où elles coexistent avec les régimes réglementaires publics et revêtent une importance croissante dans la gouvernance des filières d'approvisionnement agricoles et alimentaires. Dans une certaine mesure, la mise en place de référentiels privés pour la qualité des aliments s'explique par le poids croissant des exigences réglementaires et des risques de réputation, notamment imputable à la responsabilité du fait des produits, qui pèse sur les principales entreprises des différentes filières d'approvisionnement, et plus particulièrement les principaux détaillants et entreprises de services. Par ailleurs, cependant, ces référentiels ont permis de faciliter des stratégies concurrentielles de différenciation des produits s'appuyant sur une gamme de plus en plus large de caractéristiques ou de signes de qualité dans l'optique de répondre aux nouvelles attentes et préoccupations des consommateurs [Henson, 2006]. Les normes privées, qui sont aujourd'hui couramment employées dans divers pays développés, se propagent progressivement aux pays à revenu intermédiaire et à quelques pays à bas revenu [Henson et Reardon, 2005]. Ces évolutions reflètent en partie le mouvement de concentration qui s'est opéré dans le secteur alimentaire et se traduit notamment par une baisse du nombre des grandes chaînes de distribution et entreprises de services ainsi que des principaux fabricants. Ces sociétés détiennent un pouvoir de négociation suffisamment puissant pour imposer leurs propres normes à différents fournisseurs d'horizons géographiques très divers, de même que des stratégies concurrentielles centrées sur leurs marques propres ou des marques privées pour leurs opérations transfrontières. De ce fait, dans de nombreux pays développés, les systèmes nationaux de contrôle de la qualité des aliments correspondent de plus en plus à une juxtaposition de normes publiques et de normes privées. En dépit de leur essor, les normes privées sont donc encore loin d'être appliquées à toutes les filières, les normes publiques demeurant prédominantes dans certains pays ainsi que pour des caractéristiques et des catégories particulières de produits. 
Face au durcissement généralisé des exigences réglementaires concernant la qualité des aliments, et en réponse aux préférences changeantes des consommateurs qui, de façon générale, souhaitent une meilleure qualité et une plus grande diversité de leur alimentation, les normes alimentaires privées ne cessent d'évoluer. Non seulement les normes privées ont permis aux entreprises de relever ces défis et de différencier leurs produits, mais elles ont également eu pour effet de faire passer la concurrence sur les marchés agricoles et alimentaires d'une logique de prix à une logique de qualité. Du point de vue de la demande, le rôle des normes privées est conforté par la présence, dans les pays développés, de consommateurs aisés aux goûts raffinés et diversifiés, et du côté de l'offre, par la mise en œuvre de techniques de production, de transformation et de distribution autorisant une différenciation des produits, mais aussi une expansion et une segmentation des marchés. Comme on l'a vu précédemment, cette mutation transforme le rôle des classes et des normes, lesquelles permettent non seulement de réduire les coûts de transaction pour les acteurs des marchés agricoles, mais constituent désormais aussi des outils stratégiques que les entreprises privées utilisent aussi bien pour pénétrer les marchés, coordonner les référentiels, garantir la qualité et la sécurité de leurs produits, identifier leurs marques et définir des créneaux spécifiques [Henson et Reardon, 2005], (voir encadré 2). Dans la mesure où les normes privées permettent aux entreprises de capter les avantages des caractéristiques de qualité qu'elles offrent, elles les incitent à procéder aux investissements nécessaires pour améliorer la qualité des produits et leur fournit les moyens de motiver leurs fournisseurs par le biais de surprix et d'autres conditions préférentielles relevant de la gouvernance des filières d'approvisionnement en croissance [Henson, 2006].

Pour leur part, les consommateurs évaluent de plus en plus la qualité des produits agricoles et alimentaires en fonction d'une gamme croissante de caractéristiques, ce qui accroît d'autant l'importance des normes qui leur sont applicables. Ainsi, les consommateurs dont le niveau de revenu est élevé, exigent que les produits présentent des caractéristiques nettement supérieures, que ce soit sur le plan de leur qualité, de leur valeur nutritionnelle, de leur traçabilité santé ou de la santé [Kinsey, 2004]. Les entreprises agroalimentaires, qui évoluent dans le contexte d'un système dynamique, généralement caractérisé par de faibles marges et une inélasticité de la demande, répondent à ces attentes en intégrant les normes privées à leurs stratégies de concurrence et tentent de communiquer aux consommateurs leur conception de la qualité et de la diversité des aliments [Fulponi, 2006]. Utilisées pour renseigner les consommateurs sur les différentes caractéristiques de qualité des produits, les normes privées permettent d'accroître la fidélité des consommateurs tout en réduisant l'élasticité du prix des produits alimentaires concernés afin de récompenser les fournisseurs par des prix plus élevés pour leurs investissements entrepris dans des systèmes de gestion de la qualité [Kinsey, 2004].

Outre la différenciation des produits et la segmentation des marchés, les normes privées donnent aux entreprises les moyens de mieux coordonner ou gérer les activités de production, d'approvisionnement en matières premières et de commercialisation, ce qui leur permet de réaliser ouvrant la voie à des économies et des gains d'efficience [Holleran, 1999]. Elles peuvent ainsi entraîner une réduction des coûts de transaction, améliorer la gestion de l'offre au sein de zones géographiques étendues, éventuellement transfrontières, et standardiser les spécifications techniques imposées à un grand nombre de fournisseurs [Henson et Reardon, 2005]. En outre, le recours aux normes permet de fournir, d'identifier et de maintenir dans la durée les caractéristiques des produits et des procédés tout au long de la filière d'approvisionnement, de manière à proposer une offre qualitative permanente, confortée par des marques et une certification propres [Bergès-Sennou et al., 2004]. Cet élément est particulièrement important en ce qui concerne les caractéristiques de confiance associées aux modes de production et de manutention des produits alimentaires [Henson et Traill, 1993].

Bien que les normes privées relèvent nécessairement d'une démarche volontaire, elles peuvent être appliquées par la majorité des fournisseurs, d'où l'avantage économique que représentent la standardisation ou les exigences du marché. Pour ce qui concerne ce dernier point, les normes privées exclusives peuvent devenir quasiment obligatoires ou être «de facto » incontournables sur certains 
marchés agricoles et alimentaires, dans la mesure où les fournisseurs n'ont guère d'autre choix que de s'y conformer, soit pour entrer sur un marché contrôlé de fait par quelques grands acheteurs détenteurs d'un pouvoir d'oligopsone, soit pour pouvoir continuer d'y opérer [Henson et Northen, 1998]. Au final, la situation peut être identique à celle qui résulterait d'une réglementation publique contraignante. Ainsi, le thon « respectueux des dauphins » est une appellation relevant d'une démarche strictement volontaire, mais aux États-Unis, les boîtes de thon ne portant pas ce signe d'identification ont totalement disparu des rayons des magasins. L'application généralisée de cette norme privée en fait désormais un standard industriel. Les normes exclusives sont particulièrement répandues dans les grandes chaînes de distribution, entreprises agroalimentaires ou de services, ce qui montre bien le pouvoir de marché considérable que celles-ci détiennent et les stratégies de concurrence qu'elles ont élaborées autour de marques privées qui lient la réputation d'une entreprise au niveau de qualité des produits qu'elle offre [Henson, 2006]. Les efforts réalisés pour améliorer les normes minimales de qualité, différencier les produits et, parallèlement, gérer les coûts de transaction et les risques encourus par les grandes filières d'approvisionnement ont largement accéléré l'évolution des normes de qualité privées, plus particulièrement en Europe. On voit par là que les normes privées relèvent généralement davantage d'une démarche volontariste et agile qu'en conséquence, elles sont vraisemblablement susceptibles d'évoluer plus rapidement que les modes de régulation publique puisque les entreprises cherchent à répondre rapidement aux préférences changeantes des consommateurs. Globalement, les normes privées à caractère volontaire, qui visent à améliorer la qualité des produits alimentaires et permettent à la fois une plus grande flexibilité et une meilleure réactivité pour répondre aux souhaits des consommateurs de pouvoir disposer d'un grand choix qualitatif, sont en passe de devenir tout à la fois un élément clé de la dynamique des marchés agroalimentaires et un phénomène mondial [Henson et Reardon, 2005].

Il faut toutefois reconnaître que l'apparition relativement récente des normes privées dans les systèmes alimentaires nationaux n'est pas un phénomène isolé. En effet, si des protocoles et marques privés ont pu se mettre en place, c'est parce que, dans bien des cas, ils ont été construits en s'appuyant sur les infrastructures de normalisation existantes. De fait, ce sont les normes publiques de qualité minimales qui ont permis une différenciation qualitative des produits à un coût plus raisonnable tout en limitant les possibilités de fraude et de pratiques abusives. Aussi l'évolution des normes privées et les formes particulières qu'elles peuvent revêtir découlent-elles, en partie tout au moins, des normes publiques prédominantes dans le pays considéré. Dans d'autres cas, les normes privées ont simplement repris certaines caractéristiques des normes publiques, telles que les critères minimums à respecter en matière de sécurité alimentaire et d'hygiène des aliments, lesquels sont désormais une composante commune non concurrentielle des normes privées. Le développement des normes privées répond aussi aux modifications du niveau et de la nature des exigences réglementaires publiques. Parce que les réglementations et les normes publiques ont évolué, qu'elles sont de plus en plus strictes et que leur application se généralise, les normes alimentaires privées se sont elles aussi renforcées de manière à permettre à une entreprise agroalimentaire de continuer de différencier ses produits d'aliments sans marque plus génériques satisfaisant aux normes publiques minimales de qualité. Il en résulte que toute modification des exigences liées aux normes minimales entraîne une modification des stratégies privées de différenciation qualitative [Codron et al., 2005]. Dans d'autres cas, l'adoption de normes privées a pu être motivée par l'existence de normes publiques inadaptées. Les entreprises seront également incitées à mettre en œuvre des normes privées dès lors qu'il n'existe pas de normes publiques de qualité ou que celles-ci sont jugées insuffisantes (ou encore qu'elles ne sont pas respectées), auxquels cas les normes privées peuvent se substituer aux réglementations publiques [Reardon et al., 2001; Henson et Reardon, 2005]. Cette situation a pu être observée dans quelques pays moins développés, où les normes privées élaborées par certaines multinationales de la distribution pour leurs marchés nationaux ont également été appliquées et ont bénéficié aux consommateurs locaux [Reardon et Berdegeue, 2002]. L'adoption de normes privées peut même avoir lieu alors que les réglementations publiques offrent déjà un niveau élevé de qualité. Dans ce cas, en effet, la mise en œuvre de normes privées vise à restreindre l'exposition de l'entreprise à une responsabilité ex post du fait du produit, atténuer le risque d'atteinte à sa réputation s'il est établi qu'elle 
vend des denrées qui ne satisfont pas la norme publique de qualité minimale, ou anticiper sur des mesures réglementaires complémentaires [Lutz et al., 2000].

Compte tenu de ces diverses évolutions et contraintes, les normes privées, qui sont de plus en plus régies par les acheteurs et dont l'application se généralise peu à peu, semblent jouer un rôle essentiel dans la mutation des systèmes agroalimentaires des pays développés et, de plus en plus, des pays en développement [Henson et Hooker, 2001]. La promulgation de normes de qualité privées a été renforcée par la mise en place de métasystèmes tels que les procédures d'analyse des risques-points critiques pour leur maîtrise (HACCP), les bonnes pratiques de fabrication (BPF) ou les bonnes pratiques agricoles (BPA) [Caswell et al., 1998]. Certains observateurs considèrent ces métasystèmes comme des «codes de conduite » régissant la participation au système agroalimentaire et l'obtention d'une caractéristique de qualité spécifique [Henson et Reardon, 2005]. Ces systèmes sont de plus en plus perçus comme déterminant le fonctionnement de la filière d'approvisionnement tout entière, depuis la production agricole et la transformation jusqu'à la distribution et la vente au détail du produit final [Dolan et Humphrey, 2001 ; Fulponi, 2006]. Certains de ces métasystèmes ont été incorporés aux normes publiques volontaires applicables à l'échelle nationale ou internationale (norme ISO 22000, par exemple), tandis que d'autres correspondent à des normes privées élaborées par des organismes de normalisation (comme le référentiel SQF 2000) ou par des entreprises agroalimentaires (telles que Nature's Choice de Tesco) [Henson, 2006]. Quelques-uns d'entre eux, qui constituaient au départ des codes de bonnes pratiques relevant d'une démarche volontaire, font désormais partie intégrante des réglementations publiques, ajoutant ainsi encore à la confusion entre normes privées et normes publiques. La prise en compte du système HACCP dans les exigences réglementaires applicables à la viande et aux produits à base de viande aux États-Unis, au Canada et dans l'UE en est un bon exemple. La mise en place de normes privées peut donc constituer un moyen de se conformer aux exigences réglementaires publiques, mais il arrive aussi parfois que les réglementations publiques adoptent les mécanismes en jeu dans les normes privées et les normes de référence, tendance révélatrice des profonds changements institutionnels en cours dans un certain nombre de pays, notamment en ce qui concerne la supervision globale des filières d'approvisionnement ainsi que la qualité des aliments.

Il existe également un parallèle entre les normes alimentaires publiques et privées et leurs modes d'application respectifs. Comme les entreprises privées ne sont pas toujours en mesure de s'approprier l'intégralité des bénéfices que devrait logiquement dégager la coûteuse prise en charge du contrôle des risques liés à la responsabilité du fait des produits ou des méthodes de production, elles ne souhaitent pas nécessairement mettre en œuvre des protocoles supposés garantir que leurs produits satisfont à la norme de qualité supérieure applicable aux produits différenciés, ni procéder à un niveau de contrôle de qualité suffisant pour maîtriser les problèmes qui risquent de rendre dangereux pour la santé les produits alimentaires concernés. [Jensen et Hayes, 2006]. C'est pour remédier à cette situation que des systèmes privés d'assurance qualité se sont développés sur la base de la certification privée (à savoir auto certification, certification réciproque et certification par une tierce partie indépendante), ainsi que d'une amélioration de la transparence ${ }^{9}$ et de la traçabilité. Alors que l'autoréglementation concerne l'établissement de systèmes internes de contrôle de la qualité des produits, la certification implique un suivi et une certification de normes de produit et de procédé mesurables par une autre entreprise privée ou une tierce partie indépendante. Ce type de certification peut également correspondre à une démarche volontaire ou être stipulée par les partenaires commerciaux de l'entreprise [OMC, 2005] et varier en fonction des produits. Au vu des publications, il apparaît que la certification officielle de la qualité des produits à base de viande est très développée aux États-Unis, au Royaume-Uni, au Canada, en Australie, en Nouvelle-Zélande et au Japon. En revanche, la certification privée est très prisée dans les pays européens, mais fort peu aux États-Unis et au Japon [Jensen et Hayes, 2006]. Quelle que soit la nature de la norme

9. La notion de transparence renvoie à la fourniture d'informations sur les procédures et pratiques utilisées pour fabriquer un produit au sein de la filière de commercialisation. 
considérée, les auditeurs tiers sont généralement agréés au cas par cas par l'organisme officiel d'accréditation du pays dans lequel ils exercent leur activité, ce qui montre là encore que la crédibilité des régimes normatifs privés dépend des systèmes publics de contrôle [Henson, 2006].

Le succès rencontré par les normes volontaires privées dans le secteur alimentaire fait évoluer les processus de normalisation, notamment avec la formation de coalitions ou de consortiums d'entreprises. L'adoption de normes privées plus sévères pour la différenciation des produits et le positionnement concurrentiel sur les marchés ou pour s'assurer une protection contre la responsabilité du fait des produits a notamment conduit à une hausse des coûts de transaction pour les entreprises ayant établi leurs propres standards. Par contrecoup, ces entreprises ont réclamé la mise en place de normes privées collectives harmonisées par des organismes professionnels et des groupements d'entreprises [par exemple: référentiels British Retail Consortium (BRC) ou International Food Standards (IFS)], les normes collectives étant certifiées par un auditeur tiers [comme le European Food Safety Inspection Service (EFSIS)] ou par des entreprises privées en lieu et place des normes interentreprises [Henson et Northen, 1998]. Cette nouvelle configuration présentait l'avantage, pour chaque entreprise, de réduire les coûts d'organisation de la filière d'approvisionnement qu'elle doit supporter et d'élargir le groupe de fournisseurs potentiels auprès desquels elle pouvait s'approvisionner. En conséquence, les normes découlant d'accords globaux interentreprises (B2B) ont été étendues et se présentent désormais sous la forme de normes d'approvisionnement dont l'objectif est d'améliorer les performances globales de la chaîne de valeur et sa gouvernance [Casella 1997, 2001 ; Nadvi et Waltring, 2003 ; Fulponi, 2006]. Les normes coordonnées ou harmonisées peuvent donc garantir aux entreprises participantes un avantage concurrentiel et leur permettre de poursuivre des objectifs communs sans se concurrencer [Dolan et Humphrey, 1999; Casella, 2001]. Tandis que les sociétés d'approvisionnement et de distribution des denrées sont intéressées par une rationalisation des normes privées de marque déposée puisque leur prolifération engendrerait des coûts de transaction supplémentaires, elles craignent aussi une norme unique, gérée par un certain détaillant en aval ou société de service de restauration, sur laquelle ils n'ont aucun contrôle. Ils doivent ainsi arriver à trouver l'équilibre entre les avantages-coût d'une norme unique et la perte de liberté d'action.

Ces efforts collectifs d'harmonisation des grandes entreprises leaders se sont par ailleurs propagés des institutions nationales ou régionales (telles que le BRC et l'Euro-Retailer Produce Working Group EUREP) à des organismes internationaux privés de normalisation comme la Global Food Safety Initiative (GFSI). L'évolution des normes privées se poursuit à partir des normes interentreprises (B2B), des normes collectives et des normes de type B2C (entreprise-consommateurs). Bien souvent, les normes B2B utilisées pour soutenir et favoriser le développement de la différenciation des produits ne sont pas directement diffusées auprès des consommateurs. Néanmoins, d'autres normes de type B2C ont été mises au point par les principaux détaillants (comme la Filière agriculture raisonnée d'Auchan, la Filière qualité de Carrefour ou la Finest Brand de Tesco Stores PLC), mais également par des organisations de producteurs liées à des marques ou des logos qui sont autant de repères directs pour les consommateurs. On observe là aussi une évolution: les normes nationales deviennent des référentiels régionaux et internationaux, et les méthodes de certification et de contrôle de conformité passent du stade de l'autoréglementation à la reconnaissance mutuelle, puis par une tierce partie.

En conséquence, dans les systèmes agroalimentaires de nombreux pays, de multiples réseaux de normes publiques et privées s'enchevêtrent aujourd'hui, et cette prolifération accroît progressivement la complexité des interactions entre les différentes normes. Une tendance semble néanmoins se dessiner, en l'occurrence l'abandon progressif des normes publiques obligatoires, qui était la forme prédominante de contrôle ou de gouvernance de la qualité et des aliments, au profit de formes de contrôle volontaire avec son corollaire, le rôle de plus en plus important du secteur privé. Il suffit en effet de constater la part et la place croissantes des normes édictées à l'initiative d'entreprises ou de groupes privés, notamment des normes exclusives, qui s'imposent de facto sur les marchés alimentaires [Henson, 2006]. À mesure que le 
rôle des pouvoirs publics se réduit à la surveillance globale du système de qualité des aliments et à la supervision des performances des entreprises par le biais d'audits des systèmes de contrôle et de procédures similaires, il semblerait que beaucoup d'aspects de la qualité soient de plus en plus du ressort des normes privées [Henson et Hooker, 2001 ; Henson, 2006]. Dans certains cas, ces évolutions conduisent les acteurs publics et privés à davantage coopérer et se coordonner pour les procédures de normalisation, comme le montre le système français de normes de qualité appliquées aux fruits et légumes [Codron et al. 2005]. Il pourrait ainsi être judicieux d'explorer plus avant les approches de corégulation ou les partenariats public/privé [Garcia Martinez et al., 2005] cherchant à atteindre de manière plus efficace les objectifs de qualité des aliments.

\section{F. Normes et échanges}

La nécessité de prendre en compte les intérêts d'autres pays dans le cadre des échanges mondiaux révèle une nouvelle dimension des interactions entre normes privées et normes publiques. Il incombe avant tout aux pouvoirs publics d'adopter une politique commerciale et d'ouvrir l'accès aux marchés nationaux. Toutefois, les normes correspondant, entre autres, à des niveaux d'industrialisation et de développement économique, mais aussi à des cultures et à des valeurs nationales, elles sont susceptibles de varier d'un pays à l'autre. L'hétérogénéité qui en résulte au niveau des normes et des réglementations publiques peut à son tour avoir un impact sur les échanges internationaux. En effet, les normes influent sur la concurrence internationale, et il est désormais largement admis qu'elles peuvent constituer des obstacles non tarifaires. De plus, sous l'effet du développement des multinationales de l'agroalimentaire et de leurs pratiques d'approvisionnement planétaire, la portée des normes privées dépasse aujourd'hui le cadre des frontières nationales, de sorte qu'elles peuvent également influer sur les échanges. Les sections suivantes portent sur un certain nombre de préoccupations auxquelles est confronté le commerce alimentaire mondial en raison du caractère hétérogène des normes nationales et des normes internationales privées. Sont notamment abordées les questions suivantes: les disparités entre les normes et les réglementations nationales entraînent-t-elles une diminution des échanges et, le cas échéant, comment peut-on en minimiser l'ampleur ? Les mécanismes de l'OMC ajoutent-ils des contraintes au processus d'élaboration des normes publiques et quels sont leurs liens avec les normes privées des entreprises multinationales ? Comment des organismes internationaux de normalisation tels que le Codex Alimentarius ou l'Organisation internationale de normalisation (ISO) s'inscrivent-ils dans l'architecture des normes publiques et privées ?

\section{F.i Normes publiques et échanges}

La complexité des interactions entre les normes de qualité publiques et leurs équivalents du secteur privé augmente considérablement dans le contexte des échanges internationaux. En outre, il devient important, dans le cadre du système commercial multilatéral, de distinguer les normes imposées aux produits de celles qui concernent les procédés, en particulier lorsque ces dernières portent sur des procédés et méthodes de production (PMP) non intégrés ${ }^{10}$ [OMC, 2005]. D'un point de vue commercial, le principal risque concernant les normes publiques nationales réside dans le fait qu'elles sont préconisées par la puissance publique et qu'au cours du processus d'élaboration des normes, les pouvoirs publics peuvent ainsi privilégier les producteurs nationaux au détriment des importations en instaurant des méthodes de production et de fabrication spécifiques [OMC, 2005] susceptibles de jouer le rôle d'obstacles non

10 Si les procédés ne font généralement pas l'objet d'échanges, ce n'est pas le cas des produits qu'ils permettent d'obtenir: ils sont donc importants pour les échanges. En revanche, les PMP non intégrés n'ayant pas d'incidence directe sur les produits échangés, leur influence sur les politiques commerciales est moins évidente. Les consommateurs ou les pouvoirs publics d'un pays importateur peuvent néanmoins se préoccuper du mode de production d'une denrée importée, notamment pour prendre en compte les effets sur l'environnement du processus de production mis en œuvre (cas des crevettes aux États-Unis, par exemple). Les normes du travail possèdent enfin de nombreuses caractéristiques communes avec les normes de procédé. 
tarifaires et de restreindre les échanges. L'incidence des normes sur le bien-être peut également évoluer lorsqu'on les considère à l'aune des échanges internationaux. Comme cela a été observé à propos de la définition des normes nationales, les normes obligatoires peuvent, lorsqu'elles sont appliquées sur des marchés de biens de confiance, permettre d'améliorer l'efficience et le bien-être dans les pays concernés en éliminant les asymétries d'information entre acheteurs et vendeurs de produits alimentaires. Si une norme correctement conçue permet d'accroître le bien-être des citoyens dans le pays qui l'impose, en revanche, compte tenu de ses effets sur les échanges internationaux, elle ne se traduit pas forcément par une amélioration de l'efficience et du bien-être au niveau mondial [OMC, 2005].

Les normes nationales instaurées en vue d'accroître l'efficience sur les marchés peuvent ainsi présenter des effets complexes si l'on tient compte de leur portée commerciale [Henson, 2004]. Tout d'abord, les entreprises soumises sur leur propre marché à des normes de qualité qui diffèrent des normes en vigueur sur les marchés étrangers où elles souhaitent écouler leur production, peuvent se trouver défavorisées par rapport aux entreprises nationales. C'est également le cas des normes de production quasipubliques appliquées au niveau des exploitations. Ainsi, nombre de systèmes d'assurance qualité sont conçus et mis en œuvre par des organisations de producteurs qui ont intérêt à utiliser le dispositif à leur avantage pour différencier la production nationale des importations qui lui font concurrence [Holleran et al., 1999]. De même, les normes peuvent s'avérer contre-productives pour les exportateurs des pays dans lesquelles elles sont instaurées. Lorsque le pays établissant la norme est un pays exportateur, il est peu probable que les échanges s'y développeront. Dès lors que les coûts de production des produits alimentaires de qualité supérieure sont plus élevés, les exportateurs du pays en question, à moins d'être en mesure de différencier leurs produits et de s'assurer une majoration en raison de leur qualité, seront moins compétitifs sur le marché mondial face à des concurrents qui ne seraient pas soumis à la même norme de qualité [OMC, 2005].

Les accords commerciaux internationaux affectent également les systèmes de contrôle publics et privés [Henson et Caswell, 1999]. Ce sont a priori les normes officielles de produits et de procédés qui présentent le plus de risques pour les échanges [OMC, 2005]. Face au recul des droits de douane et des autres instruments habituellement utilisés pour la gestion des importations et le contrôle de l'accès au marché sous l'effet des accords commerciaux progressifs, subsiste la tentation de recourir aux réglementations sanitaires et phytosanitaires, ainsi qu'aux réglementations techniques pour ériger des obstacles non tarifaires dans l'optique de compenser le manque de protection des producteurs nationaux. Aussi les systèmes nationaux de réglementation des aliments, considérés comme autant d'éventuels obstacles non tarifaires aux échanges, sont-ils soumis à un examen plus minutieux, et les pouvoirs publics poursuivent-ils leurs efforts collectifs en vue d'une coordination multilatérale des activités réglementaires [Henson, 2004]. A l'échelle internationale, on s'est efforcé de remédier aux effets potentiellement négatifs sur les échanges des normes de qualité des aliments. Les accords de l'OMC sur l'application des mesures sanitaires et phytosanitaires (accord SPS) et sur les obstacles techniques au commerce (accord OTC) jouent un rôle majeur à cet égard. S'ils constituent la principale tentative visant à prendre en compte l'incidence des normes alimentaires sur les échanges, ils ont également permis des gains de transparence dans l'application de certains types de normes nationales. Ces accords confèrent désormais aux États une responsabilité officielle et les incitent à mettre bon ordre aux répercussions de leurs programmes réglementaires sur les échanges [Caswell et Henson, 1999]. Ils stipulent également que les Membres de l'OMC élaborant de nouvelles normes ou réglementations techniques non fondées sur des normes internationales doivent notifier les mesures adoptées avant leur entrée en vigueur. Le développement de ces déclarations atteste également de la multiplication et de l'impact des normes publiques.

Les règles internationales sur les produits agricoles et leurs caractéristiques alimentaires sont essentiellement définies par les accords SPS et OTC conclus en 1994. Tandis que l'accord SPS instaure des disciplines pour la réglementation des caractéristiques de sécurité des aliments, l'Accord général sur le commerce et les droits de douane (GATT)/OMC et l'accord OTC modifient les réglementations concernant 
les autres caractéristiques de qualité des aliments. L'accord OTC couvre des règlements techniques, des normes, y compris sur l'emballage, le marquage et l'étiquetage et des procédures d'évaluation de conformité. Dans le secteur agroalimentaire, l'Accord OTC s'applique à toutes les règles autres que celles touchant aux animaux, aux plantes, à la vie humaine et à la santé qui sont couvertes par de l'accord SPS. Les questions sur la qualité des aliments comme l'étiquetage nutritionnel sont couvertes par l'accord OTC. Un autre accord commercial ayant trait aux aspects des droits de propriété intellectuelle qui touchent au commerce (ADPIC) comporte des dispositions destinées à préserver l'utilisation des indications géographiques, qui permettent de distinguer les produits agricoles et alimentaires sur le marché. Après 1994, l'Organisation mondiale du commerce devient le dernier recours pour les différends opposant des Membres sur les barrières commerciales liées aux Accords SPS et OTC $^{11}$ [Kinsey, 2004].

L'accord SPS définit des principes directeurs visant à minimiser les effets de distorsion sur les échanges des normes alimentaires en vigueur qui s'appliquent à la santé et décrit brièvement les procédures de résolution des différends entre pays en la matière. Il permet notamment de justifier les normes alimentaires nationales selon deux approches : la première consiste à adopter des normes internationales systématiquement considérées comme conformes aux dispositions du ou des accord(s) et la seconde, à évaluer les risques pour la santé humaine (mais aussi pour les animaux et les végétaux) pris en compte par la réglementation alimentaire étudiée. Cet accord établit en outre un certain nombre de critères que les réglementations nationales doivent respecter, soit qu'il n'existe aucune norme internationale, soit qu'un État souhaite s'écarter ou aller au-delà d'une norme internationale donnée. L'évaluation des risques est un élément central des disciplines instaurées par cet accord pour évaluer les différentes normes. Il a également des implications majeures pour la réglementation alimentaire des différents pays concernés, avant tout en ce qu'il contraint les organismes de réglementation à adopter uniquement des contrôles de qualité fondés sur des principes scientifiques et ayant le moins d'incidences possible sur les échanges ${ }^{12}$. Les accords de l'OMC imposent des procédures très diverses, comme les obligations de notification et de consultation, ainsi que des obligations de transparence. En ce qui concerne ces dernières, il ne s'agit pas simplement d'accorder aux autres pays des délais suffisants pour formuler des commentaires sur les réglementations et pour organiser des consultations, mais d'exiger l'établissement de "points d'enquête » afin de répondre aux questions et de recevoir les commentaires d'agents d'autres États. L'accord OTC définit également des critères pour les procédures d'évaluation de conformité (comme le fait l'accord SPS pour les procédures de contrôle et d'inspection), l'objectif étant de garantir que les prescriptions de la norme seront remplies. Au vu des différends soumis à l'OMC, il est néanmoins manifeste que les autorités font l'objet de pressions internes les incitant à adopter des réglementations non conformes à ces exigences [Kinsey, 2004].

Une première évaluation des différends examinés par l'Organe d'appel de l'OMC à l'aune de ces différents accords montre que l'accord SPS a été plus efficace pour résoudre des divergences sur les réglementations relatives aux végétaux et aux animaux que pour trancher les litiges sur les mesures liées à alimentaire. L'accord SPS s'applique ainsi à toute mesure destinée à protéger la vie et la santé des humains et des animaux contre les risques de toxi-infection alimentaire; à protéger la santé humaine contre les maladies transmises par les animaux ou les végétaux; à protéger les animaux et les végétaux contre les parasites et les maladies; et à empêcher ou restreindre les dommages causés à un pays par l'arrivée, l'implantation ou la propagation des parasites. En revanche, l'accord OTC concerne l'application de toute réglementation technique, norme volontaire ou procédure d'évaluation de la conformité. Tandis que le type de la mesure permet généralement de déterminer si elle est couverte par l'accord OTC, son objectif indique si elle est ou non couverte par l'accord SPS [OMC, p. 145].

Lorsque les données scientifiques correspondantes sont insuffisantes, un Membre peut provisoirement adopter des mesures sanitaires et phytosanitaires en se fondant sur les informations pertinentes disponibles, notamment celles émanant des organisations internationales compétentes ainsi que des mesures sanitaires ou phytosanitaires appliquées par d'autres Membres. 
la sécurité alimentaire, notamment par rapport aux principes de gestion des risques [IATRC, 2001]. En revanche, l'influence de l'accord OTC sur la réglementation des autres caractéristiques de qualité des produits agricoles est plus difficile à déterminer en l'absence d'un ensemble de rapports similaires du Groupe spécial de l'OMC chargé du règlement des différends adoptés sur cette base. L'utilisation de Groupe spécial de l'OMC pour régler les différends selon l'accord OTC semble rester l'exception plutôt que la règle. L'étude de l'IATRC indique qu'en dépit d'incertitudes concernant les règles à appliquer pour ces autres caractéristiques, il existe des preuves manifestes de l'intérêt croissant des pays à l'égard des normes et des labels de qualité, perçus comme autant de moyens d'atteindre une grande diversité d'objectifs. Cette même étude constate que la plupart des efforts de réglementation dans ce domaine d'activité sont axés sur les préoccupations des consommateurs et fondés sur une acceptation croissante du principe de leur droit à l'information et à une plus grande transparence. De même que le recours à l'étiquetage pourrait être étendu dans les prochaines années à des usages plus nombreux, il semble que l'accord OTC pourrait plus largement contribuer à la réglementation des échanges internationaux de produits alimentaires [IATRC, 2001]. Ces évolutions, associées à un renforcement de la surveillance mondiale des mesures nationales, semblent indiquer que les normes réglementaires directes pourraient se généraliser progressivement à travers le monde, tant en ce qui concerne les critères appliqués que les mécanismes utilisés pour garantir leur application [Henson et Caswell, 1999].

L'effort des pouvoirs publics en vue d'atténuer les effets de distorsion sur les échanges des diverses normes de qualité nationales s'accompagne d'une volonté de coopération en matière de réglementation, désignée par le terme de «rapprochement des réglementations » [Caswell et Hooker, 1996; Hooker et Caswell, 1999]. Trois niveaux de rapprochement, variables selon le degré de coopération, peuvent être identifiés : la coordination, l'équivalence ou reconnaissance mutuelle, et l'harmonisation. La coordination renvoie aux tentatives d'atténuer les divergences entre les réglementations relatives à la qualité des produits alimentaires en vigueur dans les différents pays, notamment par l'adoption de codes de pratiques internationaux et volontaires. Le principe d'équivalence ou de reconnaissance mutuelle nécessite de reconnaître les différentes formes de normes comme étant similaires. Enfin, l'harmonisation des normes consiste à normaliser les réglementations en vigueur dans les différents pays, par exemple en adoptant des normes internationales. Une norme commune peut ainsi faciliter les échanges mondiaux en rendant les produits plus facilement substituables, en renforçant la confiance des consommateurs dans certaines caractéristiques de ces produits, en assurant la compatibilité entre produits, etc. [Henson et Caswell, 1999]. Dans la mesure où des normes nationales différentes ont segmenté le marché intérieur par rapport aux marchés étrangers, l'harmonisation devrait normalement accroître les échanges et la concurrence, réduire les prix et améliorer la qualité [OMC, 2005].

Néanmoins, les différents principes de rapprochement des réglementations ne coïncident pas toujours exactement. Dans certains cas, l'harmonisation prend le pas sur l'équivalence comme principe directeur, ou l'inverse. Dans leur précédente analyse, Hooker et Caswell [1995] indiquent que le type du produit ou de la caractéristique étudiés devrait jouer un rôle majeur dans la définition du principe directeur utilisé dans le cadre de l'OMC. Ils montrent également qu'il convient de prévoir une harmonisation des normes relatives à la sécurité des aliments et une reconnaissance mutuelle des normes de qualité. Toutefois, les arguments en faveur d'une harmonisation sont relativement peu pertinents pour les normes destinées à corriger les asymétries d'information. Compte tenu des divergences entre les pays, il semble donc préférable de disposer de plusieurs politiques et non d'une seule [OMC, 2005]. Si les accords de l'OMC ont permis certaines évolutions en termes d'impact des caractéristiques de qualité des aliments sur les échanges, les pays Membres s'opposent à une reconnaissance massive de l'équivalence des régimes réglementaires nationaux afin de s'assurer du respect du traitement national dans ce domaine ; en outre, l'harmonisation des normes s'est jusqu'ici avérée peu efficace pour l'élaboration multilatérale de normes homogènes [IATRC, 2001 ; Henson, 2004]. 


\section{F.ii Normes privées et échanges}

Outre les réglementations publiques, les normes volontaires privées sont de plus en plus connues pour leur effet potentiel sur les échanges. Les normes de qualité privées dépassent souvent largement le cadre des obligations réglementaires et peuvent donc imposer des coûts de mise en conformité substantiels aux fournisseurs et aux exportateurs. Ces coûts peuvent notamment provenir des investissements fixes liés à l'adaptation des installations de production et de transformation, des frais de personnel et de gestion induits par la mise en œuvre de la norme et des dispositifs de contrôle associés, et enfin, des coûts d'évaluation de la conformité. Ils peuvent être plus élevés pour les exportateurs de pays moins avancés en matière de normes privées et publiques [Jaffee et Henson, 2004]. En outre, étant donné les disparités potentielles entre normes volontaires de différents pays, celles-ci risquent de constituer un obstacle significatif pour les échanges ou la participation aux échanges des entreprises, groupements ou agriculteurs qui ne sont pas en mesure de se conformer aux normes d'approvisionnement [Fulponi, 2006]. Une autre préoccupation, liée au développement des normes privées, concerne leur incidence sur la transparence des processus réglementaires. Si les Membres de l'OMC sont tenus de déclarer toute nouvelle norme publique relative à la qualité des aliments, ce qui permet aux partenaires commerciaux de faire connaître leurs préoccupations et de susciter le débat, cette mesure ne s'applique pas aux normes privées [Henson, 2004]. Toutefois, la promulgation des normes privées collectives implique généralement de nombreuses parties prenantes, notamment issues de différents pays, tout au long de la filière d'approvisionnement. Ces normes pourraient donc être également exposées à l'influence d'un large éventail de participants, notamment les partenaires commerciaux [Jaffee et Henson, 2005]. La multiplication des normes collectives privées adoptées par les entreprises internationales du secteur alimentaire soulève néanmoins la question de leurs effets sur les accords SPS et OTC de l'OMC, ainsi que de la pertinence de ces normes par rapport aux droits et obligations définis dans ces accords [Jaffee et Henson, 2005]. Par exemple, le Comité SPS de l'OMC axe ses travaux sur les normes élaborées par des institutions du gouvernement central des Membres, alors que les normes privées sont principalement élaborées par des entités commerciales, si bien que le Comité n'est pas compétent pour traiter beaucoup de ces exigences ${ }^{13}$.

Le débat sur l'incidence du durcissement des normes privées sur les échanges concerne essentiellement les pays en développement et les risques qu'ils encourent de se voir exclus des filières d'approvisionnement régies par les acheteurs lorsqu'ils accèdent aux marchés des pays développés [Jaffee et Henson, 2004 ; Banque mondiale, 2005 ; Fulponi, 2006]. Ces normes sont perçues comme des barrières, soit parce que les pays en développement ne disposent pas des capacités et ressources techniques et administratives leur permettant de se mettre en conformité, soit parce que les normes peuvent être appliquées à des fins discriminatoires ou protectionnistes. Jusqu'à présent, l'analyse des incidences des normes de qualité sur les échanges a surtout été axée sur les mesures publiques et les approches essentiellement réglementaires. Bien que les travaux empiriques aient montré que les normes publiques peuvent constituer d'importants obstacles aux échanges de produits agricoles et alimentaires, on pourrait penser que les normes privées risquent d'avoir les mêmes effets, même si le corpus des recherches empiriques n'est pas comparable. Il semblerait au contraire que la tendance à l'élaboration de normes collectives privées et à leur harmonisation sur les marchés mondiaux indique plutôt qu'elles faciliteraient les échanges. Prendre en compte ces effets des normes privées sur la concurrence nécessite toutefois de reconnaître que sur de nombreux marchés agricoles et alimentaires, notamment dans le cas des céréales ou des fruits et légumes, les normes publiques restent la forme de gouvernance prédominante, si bien que les normes interentreprises (B2B) et les normes collectives privées ont peu de chances d'entraver substantiellement ces échanges [Jaffee et Henson, 2004]. D'autre part, nombreux sont les pays dont les normes privées sont moins développées, comme au Japon, et où les normes publiques continuent d'avoir une influence prédominante sur les échanges.

13

L'accord OTC, en revanche, englobe l'élaboration, l'adoption et l'application de règlements techniques et de normes par des organismes non gouvernementaux. 
Au lieu de considérer les normes traditionnelles comme des barrières, on peut les considérer comme des catalyseurs. De ce point de vue, elles stimulent les échanges des pays en développement. En effet, les nouvelles normes pourraient être utilisées par certains pays en développement pour accroître leur avantage compétitif. Elles pourraient notamment représenter une passerelle entre les exigences de plus en plus contraignantes des consommateurs et la participation de fournisseurs de pays en développement aux filières d'approvisionnement régies par les acheteurs. De fait, investir dans de nouvelles procédures pour se conformer aux normes plus strictes de ces acheteurs pourrait constituer une puissante incitation à la modernisation des filières d'exportation des pays en développement et leur permettre de se repositionner sur les marchés mondiaux concurrentiels. De plus, la conformité aux normes dans une filière peut ouvrir l'accès à de nombreuses autres filières. Le processus de mise en conformité pourrait donc être à plus long terme le fondement d'échanges plus durables et rentables. Il semble donc exister une réalité plus complexe, qui appelle une étude plus attentive de la nature précise de marchés, produits et pays particuliers pour comprendre comment l'évolution du contexte international de normalisation est porteuse à la fois de défis et d'opportunités pour les pays en développement.

Les obstacles au commerce résultant des normes privées peuvent également être éliminés grâce à une harmonisation ou une reconnaissance mutuelle. Si l'harmonisation internationale des normes volontaires peut intervenir par le biais de traités intergouvernementaux, il est plus probable qu'elle naisse de coalitions d'entreprises et de consortiums sectoriels mettant différentes normes en commun. Ces groupements sont généralement conçus pour éviter aux entreprises de multiplier les coûts de mise en conformité liés aux nombreuses normes nationales ou tout au moins de les réduire, mais aussi pour tirer parti des économies d'échelle à un niveau d'activité économique plus local [Casella, 2001]. Les pressions exercées afin d'harmoniser et de rationaliser les normes volontaires portant sur les produits alimentaires de tous les pays, ainsi que les systèmes d'évaluation de la conformité, pourraient s'accroître à mesure que les entreprises agroalimentaires se développent et mettent en place des filières d'approvisionnement à l'échelle de la planète [Casella, 2001]. Dans le même temps, il convient de reconnaître que les normes publiques conservent leur importance dans le cadre des accords interentreprises et des filières alimentaires mondiales. Avant d'accéder à un marché, les exportateurs doivent non seulement se conformer aux normes volontaires privées, mais aussi aux exigences réglementaires publiques.

\section{F.iii Normes et système commercial multilatéral}

L'un des objectifs de l'Accord du cycle d'Uruguay consistait à harmoniser plus étroitement les politiques alimentaires nationales grâce à l'adoption et au renforcement des termes du Codex Alimentarius. Si la Commission du Codex définit les normes internationales de référence en matière de qualité et de sécurité des aliments qui constituent les lignes directrices utilisées par l'OMC pour évaluer les restrictions au commerce, certains pays continuent néanmoins à fixer les règles en la matière sur leurs marchés intérieurs. Dans ce contexte, le Codex est considéré comme une première étape vers l'harmonisation des normes alimentaires mondiales [Kinsey, 2004]. En outre, les normes de la Commission économique des Nations Unies pour l'Europe (CEE-ONU), qui constituent des références internationalement reconnues, sont davantage orientées vers des préoccupations de qualité et constituent généralement la base de nombreuses classes et normes nationales. Les normes de l'OCDE relatives aux semences, aux matériels forestiers de reproduction et aux fruits et légumes entrent également pour une part importante dans ce réseau normatif intergouvernemental.

Toutefois, les études examinées relèvent plusieurs difficultés dans la capacité de la Commission du Codex à s'acquitter de cette fonction d'harmonisation. Ainsi, les objectifs définis dans l'accord SPS et visant à fonder les réglementations alimentaires sur des principes scientifiques (et non sur des besoins sociaux), s'ils permettent aux pays de définir des normes plus contraignantes que ne l'exigent les réglementations internationales pour prendre en compte les spécificités de chaque pays, notamment culturelles et alimentaires, pourraient néanmoins servir les intérêts spécifiques de ces pays [Gaull et 
Goldberg, 1993]. Une autre difficulté potentielle mentionnée par la documentation existante réside dans l'écart considérable entre les pays membres du Codex en termes de compétences et de ressources scientifiques, qui tendent à privilégier l'activité de normalisation dans les pays développés plutôt que dans les pays en développement. Si les délibérations du Codex visant à aboutir à un consensus peuvent parfois représenter une entreprise de longue haleine [Kinsey, 2004] ${ }^{14}$, ses travaux ont néanmoins permis de multiplier les normes de qualité des aliments dans le monde, les rendant indispensables pour la santé humaine et les échanges alimentaires.

Organisé par les organismes de normalisation nationaux, supranationaux ou régionaux et internationaux, le processus d'élaboration des normes évolue au fil du temps. Dans la plupart des pays industrialisés, l'infrastructure de normalisation nationale est désormais intégrée au dispositif mondial de normalisation. Ce n'est toutefois pas le cas dans tous les pays à faible revenu ou en transition, leurs organisations n'appartenant pas au réseau mondial [OMC, 2005]. Sur les 49 organismes de normalisation mondiaux recensés par une analyse de l'OMC [2005], l'Organisation internationale de normalisation (ISO) est le principal organisme non gouvernemental en charge de la qualité des produits alimentaires. Si les normes ISO sont des normes volontaires, certaines d'entre elles, mentionnées dans des réglementations techniques, notamment dans les domaines de la santé et la sécurité, comme autant d'éléments des cadres réglementaires nationaux, sont devenues de facto obligatoires. Les normes établies par l'ISO, en particulier la norme ISO 9000 sur les systèmes de management de la qualité, tendent à se concentrer davantage sur les préoccupations spécifiques des industriels, et le secteur privé y joue un rôle prépondérant. Les normes ISO ne cessent d'évoluer, la norme ISO 22000 constituant pour la première fois un référentiel mondial pour les systèmes de gestion de la qualité des aliments, qui englobe l'ensemble de la filière d'approvisionnement depuis la production jusqu'à la distribution et dépasse donc largement le champ d'application de la plupart des normes privées. Reste à savoir quelles seront les incidences de ce nouveau standard sur l'évolution future des normes privées. Par ailleurs, la certification ISO tend à devenir une obligation dans de nombreux dispositifs nationaux de normes publiques de procédés. Ceux-ci doivent également respecter les normes internationales, les procédures d'essai et de certification associées devant alors être reconnues au niveau mondial.

Les pays en développement sont de plus en plus nombreux à intégrer le système de normalisation mondial. Néanmoins, un nombre substantiel de pays à faible revenu, pourtant affectés par ce processus, n'y participent pas encore activement [OMC, 2005]. En effet, la mise en œuvre des normes et réglementations relatives aux produits alimentaires induit certains coûts, qui peuvent être élevés pour ces pays lorsque les engagements font l'objet d'une consolidation dans le cadre d'accords commerciaux négociés. Certains de ces coûts proviennent des éléments habituellement requis en termes de procédures d'essai et de certification (évaluation de conformité) pour établir qu'un produit alimentaire répond à des exigences normalisées, fondées sur un risque évalué scientifiquement. En outre, les pays en développement peuvent se trouver confrontés à une multiplication des coûts lorsqu'ils se mettent en conformité avec diverses réglementations techniques nationales pour accéder à certains marchés, par exemple ceux des différents États membres de l'Union européenne.

Dans les pays défavorisés, les organismes de normalisation font généralement partie du secteur public, la contribution du secteur privé dans ce domaine étant limitée. ${ }^{15} \mathrm{Si}$ ces pays disposent parfois de normes de

14 Dans son analyse des procédures de normalisation définies par le Codex, Kinsey cite l'exemple de la prolongation des délibérations et procédures engagées depuis 1974 par la Commission en vue d'élaborer une norme sur le parmesan. Dans d'autres cas, notamment le yaourt, les normes du Codex ont été relativement simples à définir.

Toutefois, cette situation évolue sous l'effet de la progression rapide du recours aux normes privées dans le secteur de la grande distribution et à mesure que l'industrie alimentaire se modernise [Henson et Reardon, p. 244]. 
qualité alimentaires bien documentées, l'application des normes existantes soulève un problème particulier en raison du faible niveau de ressources techniques, financières et publiques disponibles à cet effet. Lorsqu'un pays ne dispose pas des capacités suffisantes pour mettre en application et faire respecter les normes alimentaires les plus fondamentales, les normes volontaires privées et les procédures associées de gestion et de vérification peuvent fournir un dispositif complémentaire pertinent pour consolider les normes et assurer la protection des consommateurs [Henson, 2004]. Si cela permet d'améliorer la qualité des produits alimentaires et d'atteindre une certaine homogénéité entre les pays où certaines entreprises multinationales sont implantées, subsiste néanmoins le risque que ces normes ne reflètent pas entièrement les cultures et les préférences des pays en développement concernés [OMC, 2005]. Lorsque les normes privées mises en place par des entreprises présentes sur les marchés mondiaux ne permettent pas de prendre en compte des situations et des cultures spécifiques, les coûts de mise en conformité peuvent être plus élevés que dans le cas de normes élaborées au plan local [Henson, 2004 ; OMC, 2005]. Tandis que l'application de normes privées contraignantes aux exportateurs des pays en développement les expose toujours au risque de se voir exclus des marchés, avec des conséquences majeures en termes de diminution et de détournement des échanges, elle peut avoir des effets complexes sur les échanges de produits agroalimentaires [Henson, 2004]. En outre, comme il a déjà été mentionné, la progression des normes privées peut accentuer les forces et faiblesses sous-jacentes de la filière d'approvisionnement et affecter de diverses manières la position concurrentielle de certains pays en développement [OMC, 2005]. Toutefois, les exportateurs qui parviennent à accéder aux filières d'approvisionnement régies par les acheteurs peuvent également bénéficier d'avantages considérables, en termes de relations commerciales de long terme, en participant à des dispositifs basés sur des fournisseurs préférentiels.

\section{G. Quelques conclusions et domaines futurs de recherches}

De ce qui précède, il ressort que les relations entre secteurs public et privé pour l'établissement et la mise au point de normes de qualité gagnent sans cesse en complexité à mesure que ces deux types de normes se multiplient tout en devenant généralement plus strictes et diversifiées dans leurs applications, tant sur les marchés alimentaires nationaux ou que sur les marchés internationaux. Si elles sont de création relativement récente, les normes privées sont toutefois bien loin d'être appliquées dans tous les pays du globe. Dans des pays avancés, comme le Japon, les normes privées sont peu développées, tandis que les normes publiques demeurent prédominantes sur les marchés agroalimentaires.

Dans l'ensemble, les normes privées apparaissent complémentaires, plutôt que substituables, aux normes publiques pour la gestion de la qualité des aliments dans la plupart des systèmes nationaux où elles coexistent. Les normes publiques définissent les critères de qualité et les obligations de résultats, tandis que les normes privées élaborent les outils et processus de contrôle que devront appliquer les entreprises privées pour satisfaire et, fréquemment, dépasser ces normes et obligations réglementaires en raison de la concurrence existant sur les marchés caractérisés par une différenciation qualitative [Henson, 2006]. Par ailleurs, les normes privées peuvent également se substituer aux réglementations publiques, soit que cellesci soient inexistantes, soit qu'elles soient inefficaces [Reardon et al., 2001 ; Henson et Reardon, 2005]. C'est notamment ce qui s'est passé dans certains pays moins avancés, où les normes privées mises au point par certaines multinationales de la distribution pour leurs marchés nationaux, ont également été appliquées au bénéfice des consommateurs locaux [Reardon et Berdegeue, 2002].

Dans la plupart des cas, les entreprises privées cherchant à différencier leurs produits, opérer une segmentation des marchés et signaler ces changements aux consommateurs afin de les distinguer des produits alimentaires plus génériques satisfaisant aux normes publiques ont élaboré leurs propres normes privées sur le socle existant de normes publiques. Autrement dit, elles suivent une stratégie de différenciation des produits à partir d'une norme minimale publique de qualité [Codron et al., 2005]. Le niveau des normes formulées et appliquées par les pouvoirs publics influe sur celui des normes privées. À mesure que les normes publiques se durcissent, les normes privées peuvent elles aussi être plus strictes afin 
de maintenir un certain degré de différenciation des produits et leur positionnement concurrentiel sur les marchés alimentaires.

La distinction entre normes publiques et normes privées s'estompe à mesure que la complexité des normes s'accroît. De façon générale, les normes privées ont été conçues comme moyens de se conformer aux exigences réglementaires publiques, mais à l'inverse, il existe aussi des réglementations publiques qui adoptent les mécanismes employés par les normes volontaires privées, comme les dispositions HACCP, et par les normes de référence privées. Des normes privées peuvent aussi être mises en place alors même que des réglementations publiques offrent déjà un niveau élevé de qualité des produits alimentaires, afin d'anticiper sur des mesures réglementaires complémentaires [Lutz et al., 2000]. Les normes privées peuvent alors influer sur le contenu et le niveau de la réglementation publique.

La complexité des interactions entre les incitations procurées par les normes publiques de qualité et leurs équivalents du secteur privé augmente considérablement dans le contexte des échanges internationaux. Même s'il n'existe que de rares analyses empiriques des répercussions des normes alimentaires sur les échanges, celles-ci ont montré que les normes peuvent jouer aussi bien dans le sens d'une réduction que d'une augmentation des échanges de produits agricoles et alimentaires. Il ressort toutefois à l'évidence que les normes privées sont en passe de devenir un des déterminants clés de l'accès aux marchés dans certains pays industriels et qu'elles ne relèvent en aucun cas des structures de gouvernance établies par l'OMC et n'entrent pas non plus dans le cadre des accords SPS et OTC [Henson, 2006].

Si l'on considère le système de normalisation dans son ensemble, on observe qu'il subsiste de nombreux cas où, en dépit des mesures qu'ils prennent pour satisfaire à toute une série de normes, les acteurs du marché peuvent encore être confrontés à des inefficiences susceptibles d'amoindrir la cohérence et l'efficacité globales du système [Valceschini et Saulais, 2005]. C'est notamment le cas des normes privées exclusives mises en place par des détaillants et des entreprises de services, dont les normes ne reposent sur aucun processus consensuel au niveau sectoriel. Chaque entreprise met en œuvre ses propres normes volontaires qui, tout en étant similaires, ne sont pas nécessairement identiques et peuvent définir des exigences différentes selon les fournisseurs, généralement sur la base d'accords contractuels. Cette diversité peut générer des surcoûts et des inefficiences pour les fournisseurs qui doivent se conformer à différentes normes clients existant dans les filières d'approvisionnement. Ce type de problème peut également se poser pour les centrales d'achat privées appliquant des normes exclusives et qui sont soumises à diverses réglementations nationales ou internationales dont les dispositions peuvent différer (à titre d'exemple, les exigences HACCP sont exclues en France pour les produits agricoles non transformés, mais elles leur sont applicables au titre du Codex Alimentarius). Les protocoles collectifs privés et les normes alimentaires internationales de type système (comme la norme ISO 22000) constituent des solutions envisageables pour améliorer la cohérence globale du système de normes alimentaires, mais il n'en existe pas toujours ou bien ils ne sont pas systématiquement adoptés.

Cette synthèse de la littérature disponible sur les normes alimentaires publiques et privées pourrait suggérer que la recherche sur la manière dont ces normes interagissent dans le règlement des marchés alimentaires nationaux et internationaux est un domaine de travail relativement nouveau, mais qui reçoit une attention croissante. Il apparait que des différences existent entre les États membres de l'OCDE sur la manière dont les systèmes de normes alimentaires se développent et sur les rôles respectifs joués par des agences privées et publiques dans ce processus. Des recherches futures pourraient se concentrer sur la comparaison des gestions de la qualité alimentaire dans les principaux pays et les différents rôles joués par les secteurs publics et privés dans les aspects clefs du processus tels que par exemple la certification de qualité d'un produit et les exigences de conformité des tests. L'objectif serait d'identifier les similarités et les différences d'approche et de mettre en évidence les raisons de cela et les principaux facteurs impliqués. 


\section{BIBLIOGRAPHIE}

Akerlof, G. (1970). The Market for lemons: Qualitative uncertainty and the market mechanism. Quarterly Journal of Economics, 84: 1, pp. 488-500.

Andersen, E. S., et Philipsen, K. (1998). The Evolution of credence Goods in Consumer Markets: Exchanging Pigs in Pokes. Draft Working Paper.

Antle, J. M. (1995). Choice and Efficiency in Food Safety Policy, The AEI press, Washington, DC.

Banque mondiale (2005). Food safety and Agricultural Health Standards: Challenges and Opportunities for Developing Country Exports, Report No. 31207, Poverty Reduction \& Economic Management Trade Unit and Agriculture and Rural Development Department, Banque mondiale, Washington, DC.

Becker, T. (1999). The Economics of Food Quality Standards. Proceedings of the Second Interdisciplinary Workshop on Standardization, Hambourg, Allemagne, mai.

Bergès-Sennou, F., Bontems, P., et Réquillart, V. (2004). Economics of Private labels: A Survey of Literature. Journal of Agricultural and Food Industrial Organisation. 2:3, pp. 1-23.

Bocksteal, N. E. (1984). The Welfare Implications of Minimum Quality Standards. American Journal of Agricultural Economics, vol. 66, pp. 466-471.

Boom, A. (1995). Asymmetric International Minimum Quality Standards and Vertical Differentiation. Journal of Industrial Economics, XLIII: 1, pp. 101-119.

Bowbrick, P. (1992). The Economics of Quality, Grades and Brands. Londres et New York, Routledge.

Blumenthal, G. (2006). Quality and Food safety Impacts on Competitiveness. World Perspectives, Inc., vol. $18 \mathrm{n}^{\circ} 6$, Washington, DC.

Brunsø, K., Fjord, T. A, et Grunert, K. G. (2002). Consumers' Food Choice and Quality Perception. MAPP working paper 77, Aarhus School of Business, Aarhus.

Bureau, J-C. (2004). Raising the Bar on Product and Process Standards: Economic Principles. Banque mondiale, Washington, DC.

Buzby, J. C, et Frenzen, P. D. (1999). Food Safety and Product Liability. Food Policy, vol. 24, pp. 637-651.

Buzby, J. C. et Crutchfield, S. R. (1999). New Regulation Juice Underway, Food Review vol. 22 (2), pp 23-25.

Casella, A. (1996). Product Standards Coalitions in a Market Without Borders. NBER Working Papers 5853.

Casella, A. (1997). Free trade and Evolving Standards, in Bhagwati, J. N, et Hudec, R. E. (dir. publ.), Fair Trade and Harmonisation, Prerequisites for Free Trade? MIT Press, Cambridge, Mass. 
Casella, A. (2001). Product Standards and International Trade: Harmonisation Through Private Coalitions? Kylos, 54 2/3, pp. 243-264.

Caswell, J. A., et Henson, S. J. (1999). Interaction of Private and Public Food Quality Control Systems in Global Markets, in Loader, R. J, Henson, S. J, Traill, W. B. (dir. publ.), The Proceedings of Globalisation of the Food Industry: Policy Implications. University of Reading, Reading, Royaume-Uni, pp. 217-235.

Caswell, J. A., et Hooker, N. H. (1996). HACCP as an International Trade Standard. American Journal of Agricultural Economics, vol. 78 (3), pp. 775-779.

Caswell, J. A., Bredahl, M. E., et Hooker, N. (1998). How Quality Management Metasystems are Affecting the Food Industry. Review of Agricultural Economics, vol. 20 (2), pp. 547-557.

Clayton, K. C., et Preston, W. P. (2003). The Political Economy of Differentiating Markets: Facing Reality Inside the US Department of Agriculture. American Journal of Agricultural Economics, vol. 85(3), pp. 737-741.

Codron, J-M., Fares, M., et Rouvière, E. (2005). De la régulation publique à une régulation privée? Les conventions d'autocontrôle sur les marchés d'importation de fruits et légumes, International Journal of Agricultural Resources, Governance and Ecology, Vol. 6, No.3 pp. 415 - 427.CNUCED (2001). Food Quality Standards Definitions and Role in International Trade, Rapport du Secrétariat de la CNUCED, UNCTAD/DITC/COM/34, décembre.

Crampes, C., et Hollander, A. (1995). Duopoly and Quality Standards. European Economic review, vol. 38, pp. 71-82.

Cropper, M. L., et Oates, W. E. (1992). Environmental Economics: A Survey. Journal of Economic Literature, vol. 30, pp. 675-740.

Darby, M. R., et Karni, E. (1973). Free Competition and the Optimal Amount of Fraud. Journal of law and Economics, vol. 16, pp. 67-88.

David, P. A. (1985). Clio and the Economics of QWERTY, American Economic Review, Papers and Proceedings 72 (2).

Dolan, C., et Humphrey, J. (2001). Governance and Trade in Fresh Vegetables: The Impact of UK Supermarkets on the African Horticulture Industry. Journal of International Economics, vol. 3, pp. 111-125.

Ecchia, G., et Lambertini, L. (1997). Minimum Quality Standards and Collusion. Journal of Industrial Economics, vol. 45, pp. 101-113.

Farrell, J., et Saloner, G. (1985). Installed Base and Compatibility: Innovation, Product Pre-announcement and Predation. American economic review, vol. 76, pp. 940-955.

Ferrier, P., et Lamb, R. (2007). Government Regulation and Quality in the US Beef Market. Food Policy 32, pp. 84-97.

Flynn, A, Yakovieva, N., Green, K., et Marsden, T. (2003). Consumption and policy: the case of food. 
Fulponi, L. (2006). Final report on private standards and the shaping of the agro-food system, Working Party on Agricultural Policies and Markets, OCDE.

Gal-Or, E. (1989). Quality and Quantity Competition. The Bell Journal of Economics, vol.43, pp. 590-600.

Garcia, L. D., Wallman, K., Leickly, B., et Willey, S. (2004). Public and Private Interests in Standard Setting: Conflict or Convergence, The Standards Edge, Ann Arbor.

Garcia Martinez, M., Fearne, A., Caswell, J., et Henson, S. (2007). Co-regulation as a Possible Model for Food Safety Governance: Opportunities for Public-Private Partnerships, Food Policy 32, pp. 299-314.

Gardner, B. (2003). US food Quality Standards: Fix for Market Failure or Costly Anachronism? American Journal of Agricultural Economics, août, pp. 725-730.

Gaull, G. E., et Goldberg, R. A. (1993). The Emerging Global Food System: Public and Private Sector Issues, Wiley publishers.

Gonzalez-Diaz, M., et Raynaud, E. (2004). The Diversity of Product Quality Enforcement Devices and their Interactions, SEC03-05334, avant-projet.

Grunert, K. G. (2005). Food quality and safety: consumer perception and demand. European Review of Agricultural Economics, vol. 32 (3), pp. 369-391.

Hall, W. (1999). Issues and Activities Involved in Agricultural Grades and Standards, USDA Agricultural Marketing Service.

Harrison, M., Flynn, A., et Marsden, T. (1997). Contested regulatory practice and the implementation of food policy: exploring the local and national interface; Transactions of the Institute of British Geographers.

Henson, S. (2004). Standards and Trade: An Overview, Department of Agricultural Economics \& Business, University of Guelph, Ontario, Canada.

Henson, S. (2006). The Role of Public and Private Standards in Regulating International Food Markets. Paper prepared for the IATRC Summer Symposium Food regulation and trade: Institutional framework, concepts of analysis and empirical evidence. Bonn, Allemagne.

Henson, S., et Caswell, J. (1999). Food safety regulation; an overview of contemporary issues, Food Policy 24(6), pp. 589-603.

Henson, S. J., et Hooker, N. J. (2001). Private sector management of food safety: public regulation and the role of private controls. International Food and Agribusiness Management Review, vol. 4, pp. 7-17.

Henson, S., et Loader, R. (1995). Contemporary Food Policy Issues and the Food Supply Chain. European Review of agricultural Economics, 22 (3).

Henson, S. J., et Northen, J. R. (1998). Economic determinants of Food Safety Controls in the Supply of Retailer Own-Branded Products in the UK. Agribusiness, vol. 14 (2), pp. 113-126. 
Henson, S. J., et Reardon, T. (2005). Private Agri-food Standards: Implications for Food Policy and the Agri-food System. Food Policy, vol. 30, pp. 241-253.

Henson, S., et Traill, B. (1993). The Demand for Food Safety, Market Imperfections and the Role of Government. Food Policy, vol. 18(2), pp. 152-162.

Hill, L. D. (1990). Grain Grades and Standards: Historical issues shaping the future, University of Illinois Press.

Hirshleifer, D., et Welch, I. (1998). Learning from the Behaviour of Others; Conformity, Fads and Informational Cascades. Journal of Economic Perspectives, vol. 12, pp. 151-170.

Hobbs, J. E., et Kerr, W. A. (1992). Costs of monitoring food safety and vertical coordination in agribusiness: what can be learned from the British Food safety Act 1990? Agribusiness, vol. 8 (6), pp. 575-584.

Holleran, E., Bredahl, M. E., et Lokman, Z. (1999). Private Incentives for Adopting Food Safety and Quality Assurance. Food Policy, vol. 24, pp. 669-683.

Hooker, N. H. et Caswell, J. A. (1999). A Framework for Evaluating Non-tariff Barriers to Trade Related to Sanitary and Phytosanitary Regulations. Journal of Agricultural Economics, vol. 50 (2), pp. 152-162.

IATRC (2001). Agriculture in the WTO. The Role of Product Attributes in the Agricultural Negotiations. The International Agricultural Trade Research Consortium. Commissioned Paper No. 17, Washington, DC.

Ippolito, P. M. (2003). Asymmetric Information in Product Markets: Looking at Other Sectors for Institutional Approaches. American Journal of Agricultural Economics, vol. 85(3), pp. 731-736.

Jaffee, S., et Henson, S. J. (2004). Standards and Agri-food Exports from Developing Countries: Rebalancing the Debate, World Bank. Policy research Working Paper 3348, Banque mondiale, Washington, DC.

Jensen, H. H., et Hayes, D. J. (2006). Private Sector Approaches to Secure Traceability, Transparency and Quality Assurance in Food Chains, IATRC Summer symposium, Bonn, Allemagne, 23-30 mai.

Jones, E., et Hill, L.D. (1994). « Re-engineering Marketing Policies in Food and Agriculture: Issues and Alternatives for Grain Grading Policies », in D.I. Padberg (dir. publ.), Re-Engineering Marketing Policies for Food and Agriculture, Food and Agricultural Marketing Consortium, FAMC 94-1, Texas A\&M.

Josling, T., Roberts, D., et Orden, D. (2004) Food Regulation and Trade: Towards a Safe and Open Global System, Institute for International Economics, Washington, DC.

Katz, M. L., et Shapiro, C. (1985). Network Externalities, Competition and Compatibility. American Economic Review, vol. 75, 3, pp. 424-440.

Kindlberger, C. P. (1983). Standards as Public, Collective and Private Goods. KTKLOS, vol. 36, pp. 377-396. 
Kinsey, J. (2004). Food Safety and Security Issues in Agro-food Policy and Global Trade. University of Minnesota, Minnesota.

Lancaster, K. (1966). Consumer Demand: A New Approach. New York, NY Columbia University Press

Leland, H. E. (1979). Quacks, Lemons and Licensing: A Theory of Minimum Quality Standards. The Journal of Political Economy, vol. 87:6, pp. 1328-1346.

Loader, R., et Hobbs, J.E., Strategic Responses to Food Safety legislation, Food Policy 24 (6), pp. 625-635.

Lutz, S., Lyon, T. P., et Maxwell, J. (2000). Quality leadership when regulatory standards are forthcoming. The Journal of Industrial Economics, XLVIII, pp. 331-348.

Magat, W. A., et Viscusi, W. K. (1992). Informational Approaches to Regulation. Cambridge, MA. MIT Press.

Marette, S., Bureau, J.-C., Coestier, B., et Gozlan, E. (2003). La réglementation de la sécurité alimentaire au sein de l'Union européenne, UMR Economie publique, INRA-INAPG, Grignon.

Matutes, C., et Regibeau, P. (1996). A Selective Review of the Economics of Standardization - Entry Deterrence, Technological Progress and International Competition. European Journal of Political Economy, vol. 12, pp. 193-209.

McCluskey, J. (2006). Pre-empting public Regulation with Private Food Quality Standards, IATRC Summer symposium, Bonn, Allemagne, 28-30 mai.

Mortimore, S., et Wallace, C. (1998). HACCP: A Practical Approach. Aspen Publishers, Gaithersburg, MA.

Nadvi, K., et Waltring, F. (2003). Making Sense of Global Standards. In Schmitz, H. (dir. publ.) Local Enterprises in the Global Economy: Issues of Governance and Upgrading. Elgar: Cheltenham.

Nelson, P. (1970). Information and Consumer Behaviour. Journal of Political Economy, vol. 78, pp. 311-321.

OMC (2005). Analyse des liens entre le commerce, les normes internationales et l'OMC, Rapport sur le commerce mondial, Organisation mondiale du commerce, Genève.

Raikes, O., Jensen, M. F., et Pontwe, S. (2000). Global commodity chain analysis and the French filière approach: comparison and critique. Economy and Society, vol. 29, pp. 390-417.

Reardon, T., Codron, J.-M., Busch, L., Bingen, J., et Harris, C. (2001). Global Change in Agri-Food grades and Standards; Agribusiness Strategic Responses in Developing Countries. International Food and Agribusiness Management Review, 2(3/4), pp. 421-435.

Reardon, T., et Berdegeue, J. A. (2002). The rapid rise of supermarkets in Latin America: Challenges and Opportunities for Development. Development Policy Review, vol. 20 (4), pp. 371-388.

Reardon, T., et Farina, E. (2002). The rise of private food quality and safety standards: illustrations from Brazil. International Food and Agribusiness Management Review, vol. 4 (4), pp. 413-421. 
Ronnen, U. (1991). Minimum Quality Standards, Fixed Costs and Competition. The Rand Journal of Economics, vol. 22:4 pp. 490-514.

Segerson, K. (1998). Mandatory vs. Voluntary Approaches to Food Safety. Research Report No. 36, Food Marketing Policy Center, Department of Agricultural and Resource Economics, University of Connecticut, Connecticut.

Shapiro, C. (1983). Premiums for High Quality Products as Returns to Reputation. The quarterly Journal of Economics, vol. 98. pp. 659-679.

Shy, O. (1995). Industrial Organisation: Theory and Applications. Cambridge, Mass, The MIT Press.

Sunding, D. L. (2003). The Role for Government in Differentiated Product Markets: Looking to Economic Theory. American Journal of Agricultural Economics, août, pp. 720-724.

Sykes, A. O. (1995). Product Standards for Internationally Integrated Goods Markets. The Brookings Institution, Washington, DC.

Unnevehr, L. J., et Jensen, H. H. (1996). HACCP as a Regulatory Innovation to Improve Food Safety in the Meat Industry. American Journal of agricultural Economics, vol. 78 (30), pp. 764-769.

Unnevehr, L. J. and Jensen, H.H., (1999). The Economic Implications of using HACCP as a Food Safety Regulatory Standard. Food Policy 24(6) pp 625-635.

Valceschini, E., Saulais, L., et Barrey, S. (2005). Articulation entre réglementation, normalisation et référentiels privés dans les industries agroalimentaires. Rapport final, rapport d'une étude financée par le ministère de l'Agriculture et de la Pêche - Direction des Politiques Économique et Internationale (référence MAP 05 D1 05 01).

Venturini, L. (2002). Public Regulation and Voluntary Approaches to Food Safety for Credence Goods.

Verbeke, W. (2005). Agriculture and the Food Industry in the Information Age. European Review of Agricultural Economics, vol. 32(3), pp. 347-368.

Viscusi, W. K. (1989). Towards a Diminished Role for Tort Liability, Social Insurance, Government Regulation and Contemporary Risks to Health and Society. Yale Journal on Regulation, vol. 6 (1), pp. 65-107.

Viscusi, W. K. (1985). Consumer Behaviour and the Safety Effect of Product Safety Regulation. The Journal of Law and Economics, vol. 27, pp. 527-553.

Young, L. M., et Hobbs, J. E. (2002). Vertical Linkages in Agri-Food Supply Chains: Changing Roles for Producers, Community Groups and Government Policy. Review of Agricultural Economics 24, pp. 428-441. 